\title{
Lagrangian approach to geostrophic adjustment of frontal anomalies in a stratified fluid
}

\author{
R. PLOUGONVEN* and V. ZEITLIN \\ Laboratoire de Météorologie Dynamique, Ecole Normale Supérieure, \\ 24 rue Lhomond, 75231 Paris Cedex 05, France
}

(Received 11 September 2003; in final form 1 June 2004)

\begin{abstract}
Geostrophic adjustment of frontal anomalies in a rotating continuously stratified fluid is studied in the standard framework of strictly rectilinear fronts and jets. Lagrangian approach to this problem is developed allowing to analyze, in a conceptually and technically simple way, both major problems of the nonlinear adjustment: the existence of a smooth adjusted state for a given set of initial conditions and the attainability of the adjusted state during the adjustment process. Dynamical splitting into balanced (adjusted state) and unbalanced (inertia-gravity waves) motions becomes transparent in the Lagrangian approach. Conditions of existence of the balanced state in the unbounded domain are established. It is shown that nonexistence of a smooth adjusted state in the vertically bounded domains is generic and a parallel with the classical scenario of deformation frontogenesis is developed. Small perturbations around smooth adjusted states are then studied with special emphasis on the wave-trapping inside the jet/front. Trapped modes with horizontal scales comparable to the width of the jet are explicitly constructed for a barotropic jet and their evolution is studied with the help of the WKB-approximation for weakly baroclinic jets. Modifications of the standard scenario of adjustment due to subinertial (quasi-) trapped modes and implications for data analysis are discussed.
\end{abstract}

Keywords: Frontogenesis; Geostrophic adjustment

\section{Introduction}

Geostrophic adjustment is the process of relaxation of atmospheric and oceanic perturbations to a geostrophically balanced state where the pressure force and the Coriolis force are in equilibrium. It is well-known that synoptic-scale motions in the atmosphere and the ocean are (almost) balanced and that adjustment on a timescale that is fast relative to the inertial timescale $\left(f^{-1}\right.$, where $f$ is the Coriolis parameter), takes place when a region of imbalance arises in the fluid. Examples of phenomena

* Corresponding author. Present address: Mathematical Institute, University of St Andrews, St Andrews KY16 9SS, Scotland, UK. E-mail: riwal.plougonven@polytechnique.org 
at the origin of imbalance are diabatic heating, localized mixing due to small-scale turbulence, or forcing of the surface of the ocean by the wind.

The standard setting for adjustment studies was laid in the pioneering paper by Rossby (1938) and consists in studying the relaxation of strictly rectilinear fronts/jets neglecting variations in the along-front direction. The classical scenario of geostrophic adjustment, cf (Gill, 1982), is based on the linear approximation and is essentially as follows: a balanced part in the initial state can be identified using the linearized conservation laws; the rest of the initial anomaly consists of waves, which propagate away rapidly, i.e. in a few inertial periods. An example of this linear approach for jet-like anomalies which we are interested in may be found in the work of Fritts and Luo (1992) who calculated how the initial perturbation projects onto a balanced part and gravity wave modes, respectively. The frequencies of the excited waves are controlled by the spatial dimensions of the initial perturbation: for a jet-like anomaly, low-frequency inertia-gravity waves are excited and propagate away symmetrically with respect to the jet axis.

The problem of fully nonlinear geostrophic adjustment, however, has not received much attention in spite of its fundamental importance. Its solution would provide an answer to the conceptual question on the possibility and feasibility of separation of an arbitrary flow into a slow, balanced part and a fast part, and the (non) interaction between them (the problem of the slow manifold in geophysical fluid dynamics). More practically, identification and quantification of non-orographic sources of inertia-gravity waves in the atmosphere and ocean is directly related to the adjustment problem.

The full problem being prohibitively complex, a reduced diagnostic approach to geostrophic adjustment of strictly rectilinear fronts was developed in a number of works (e.g. Ou, 1984; Blumen and Wu, 1995; Kalashnik, 1998) following the ideas of Rossby (1938). It consists in finding a tentative final adjusted state as a stationary state with the same values of the conserved quantities (potential vorticity and geostrophic momentum, see below) as the initial state. Examples of non-existence of a smooth adjusted state for given initial conditions were thus found. The transient part of the flow (i.e. the relaxation process itself) is out of reach of such approach. The adjusted state realizes an energy minimum within the class of states with the same values of the conserved quantities and the conjecture that the system reaches it in a dissipation-less way, by emitting inertia-gravity waves, is plausible. However, the dissipation may be forced, in the strongly nonlinear case, e.g. by wave-breaking. The breaking events may change the distribution of otherwise conserved quantities (in particular the potential vorticity (PV)) and therefore 'deviate' the adjustment process. Also, the assumption that the inertia-gravity waves quickly propagate away is based on the linear theory of gravity waves in a fluid at rest: the effect of the jet on their propagation needs to be considered. Thus, the two major problems of fully nonlinear adjustment are

- the existence of the smooth adjusted state,

- the attainability of the adjusted state and the role of nonlinear wave dynamics during the relaxation process.

In the simplest rotating shallow-water model, the fully nonlinear geostrophic adjustment of flows having no variations in one direction was revived recently after the numerical work by Kuo and Polvani (1997). Their experiments showed in particular 
that the adjustment process could be retarded. They also found that shock formation is typical for the nonlinear adjustment. The fully nonlinear adjustment of rectilinear fronts in the rotating shallow-water model was then examined analytically in Zeitlin et al. (2003), where advantages of the Lagrangian approach to the problem were demonstrated, in particular while describing the separation of the flow into a balanced and a fast part, and in understanding shock formation. An important result was a demonstration of existence and uniqueness of the adjusted state for localized initial anomalies with positive potential vorticity. This work was continued in the numerical study of fully nonlinear adjustment in rotating shallow water undertaken in Bouchut et al. (2004). By using high-resolution shock-capturing numerical methods, it was confirmed that wave-breaking and shock formation are ubiquitous and frequently happen within the jet/front core. At the same time, it was shown that these processes, although dissipating energy efficiently, cannot change the PV of strictly rectilinear configurations in rotating shallow water. The relaxation toward a well-defined adjusted state was observed in all cases, including non positive-definite PV distributions, although slowly dispersing quasi-inertial oscillations were systematically observed in the vicinity of the front.

Density and temperature stratification, which are obviously absent in the rotating shallow water model, are key actors in atmospheric and oceanic fronts. Geostrophic adjustment of localized anomalies having no variation in the along-front direction has been studied in the stratified fluid in some particular cases by a number of authors. Flat bottom and rigid lid boundary conditions were generally imposed, and various sets of dependent and independent variables were used. One of the motivations for these studies came from the observation of density fronts appearing due to differential heating in the coastal regions (see e.g. Csanady, 1971). Ou (1984) calculated the adjusted state for an initially motionless fluid with a horizontal gradient of density having zero PV. He showed in particular that if the initial density gradient was too intense, a continuous adjusted state no longer existed. As in the classical deformation frontogenesis scenario (Hoskins and Bretherton, 1972), the loss of continuity in the adjusted state appeared on the boundary. Adjustment of zero-PV fluid was revisited by $\mathrm{Wu}$ and Blumen (1995) for the case where the fluid is not initially motionless but has a barotropic shear. They obtained indications that the presence of shear could change substantially the adjusted state. Blumen and $\mathrm{Wu}$ (1995) have analyzed the adjustment of an initially motionless fluid with uniform PV between the flat bottom and the rigid lid, using a change of variables that had been previously used by Hoskins and Bretherton (1972). Here again, it was shown that if the initial density gradient was strong enough, no continuous adjusted state existed. Kalashnik (1998) reconsidered the problem and proposed a way to define a discontinuous adjusted state when the initial density gradient is too strong. He carried out a similar analysis for the case of a vertically semi-infinite stratified fluid (Kalashnik, 2000) and interpreted the results as spontaneous frontogenesis (in contradistinction with the deformation frontogenesis).

As was already said, an important question not addressed in the diagnostic approach is that of the dynamics of the fast part of the flow, and its interaction with the adjusted state. For strictly rectilinear configurations only waves transverse to the front are possible (no Doppler effect). The transverse waves excited by the geostrophic adjustment of a rectilinear jet-like anomaly have been studied in Fritts and Luo (1992) but no influence of the jet on the waves was taken into account. It is known, however, 
that jets may alter considerably the propagation of transverse waves, and can even trap near-inertial waves in the region of the anticyclonic shear. Yet, the implications of this trapping in anticyclonic regions have not been discussed in the context of the geostrophic adjustment problem.

Wave-trapping has been addressed in the oceanic context, starting with the WKB study by Kunze (1985) for rectilinear jets. It has also been investigated numerically (e.g. Klein and Tréguier, 1995; Xing and Davies, 2004) and by data analysis (e.g. van Haren, 2003) with special emphasis on near-inertial waves. Trapping of subinertial waves in anticyclonic regions of the flow has also been studied theoretically for axisymmetric vortices (Kunze and Boss, 1998; Llewllyn-Smith, 1999). Kunze (1985) gave results for waves that have small horizontal scales relative to the scale of the jet. However, low-frequency waves commonly have large horizontal scales, and it is desirable to go beyond the assumption of a separation between the horizontal scale of the jet and that of the waves, especially in the context of adjustment of the jet-like anomalies.

Based on the advantages brought in by the use of Lagrangian variables in the case of rotating shallow water (cf. Zeitlin et al., 2003), we propose below a Lagrangian approach to the problem of nonlinear adjustment in the continuously stratified fluid. This approach allows us to obtain new results both on existence of the adjusted state and its attainability. It provides a remarkably efficient shortcut for derivation of all of the old results in a unified way. The main advantage of this approach is that separation of flows into a balanced and an unbalanced component, which is obscured by the effects of advection in Eulerian variables, becomes trivial in the "two-and-a-half"dimensional geometry of rectilinear fronts. As in the rotating shallow water, the Lagrangian approach gives here, for adjustment in an unbounded domain, the same criterion of positivity of PV for the existence of the adjusted state. It allows to pinpoint the obstacles for the proof of existence in the bounded domains. Moreover, it makes it possible to establish a parallel between the full adjustment problem and the semigeostrophic description of the frontogensis, elucidating the relationship between the spontaneous and classical deformation frontogenesis. The approach is also efficient in treating the problem of incomplete adjustment due to trapping of sub-inertial waves. We derive a generalised prognostic Sawyer-Eliassen equation for ageostrophic motions on the background of a balanced state. We extend here the previous analyses to waves having a horizontal scale similar to that of the jet which gives a more accurate estimate of the lower bound for the waves' frequencies, and provides description of the full horizontal structure of the waves.

The plan of the article is as follows. In section 2, the Lagrangian formulation of the adjustment problem is given. We analyze the problem of diagnostic adjustment in section 3. We first establish a general criterion of existence of the adjusted state in the unbounded domain and then identify the obstacles for the proof of existence in the vertically bounded domains. Finding adjusted states from the initial conditions in traditional cases of fluids with zero or uniform PV is also described in this section. Dynamics of the fast part of the flow and the problem of attainability of the adjusted state are investigated using a perturbative approach in section 4 . We first establish and analyze the corresponding linearized equations of motion and then demonstrate how wave-trapping is happening within a vertically bounded barotropic jet and discuss the related alterations of the classical scenario of geostrophic adjustment. Propagation of the waves in a baroclinic jet varying slowly in the vertical is also considered in this 
section. Summary and discussion are presented in section 5. In appendix A, we show that the semi-geostrophic deformation frontogenesis by Hoskins and Bretherton (1972) is equivalent to a succession of diagnostic geostrophic adjustment problems; the implications of this fact are discussed. Appendix B contains an explicit construction of trapped modes in a jet with piecewise-linear vorticity profile. The consequences of the wave-trapping for practically important Eulerian diagnostics of the flow are considered in appendix $\mathrm{C}$.

\section{Lagrangian approach to the frontal adjustment}

\subsection{Primitive equations and invariants of motion}

We use inviscid, Boussinesq, hydrostatic and adiabatic primitive equations (cf. Hoskins and Bretherton, 1972; McWilliams and Gent, 1980); for consistency of notation, we denote the Eulerian coordinates by $(X, Y, Z)$ :

$$
\begin{aligned}
\frac{\mathrm{D} \mathbf{u}_{H}}{\mathrm{D} t}+f \mathbf{e}_{Z} \times \mathbf{u}_{H}+\nabla_{H} \phi & =\mathbf{0}, \\
\frac{\partial \phi}{\partial Z} & =g \frac{\theta}{\theta_{r}}, \\
\nabla_{H} \cdot \mathbf{u}_{H}+\frac{\partial w}{\partial Z} & =0, \\
\frac{\mathrm{D} \theta}{\mathrm{D} t} & =0,
\end{aligned}
$$

where $\phi$ is the geopotential, $g$ is the gravitational acceleration constant and $\theta_{r}$ is a reference value for the potential temperature. The index $H$ means horizontal part $(X, Y)$. The meaning of $Z$ and $\theta$ is different for the oceanic and atmospheric applications. In the oceanic context, $Z$ is physical height and $\theta$ is density up to a sign. For the atmospheric interpretation of equations $(1 \mathrm{a}-\mathrm{d}), \theta$ is potential temperature, and $Z$ is the modified pressure coordinate first introduced in Hoskins and Bretheton (1972) (pseudo-height):

$$
Z=Z_{a}\left[1-\left(\frac{P}{P_{r}}\right)^{(\gamma-1) / \gamma}\right]
$$

with $Z_{a}=[r /(r-1)] P_{r} / \rho_{r} g$, and $\gamma=c_{p} / c_{v}$. In these definitions, $P_{r}$ and $\rho_{r}$ are surface pressure and density constants, $c_{v}$ and $c_{p}$ are the specific heats of air at constant volume and constant pressure respectively. The pseudo-height $Z$ and the physical height $h$ are related as follows: $\theta \mathrm{d} Z=\theta_{r} \mathrm{~d} h$.

We will extensively use the Lagrangian invariants of the equations (1a-d). Two standard invariants of the equations of motion are potential temperature $\theta$ and the potential vorticity (PV), defined as $q=\left(f \mathbf{k}+\nabla \times \mathbf{u}_{H}\right) \cdot \nabla \theta$ (vertical velocity is absent because of the hydrostatic approximation). In what follows we consider configurations 
with no variation in the along-front $(Y)$ direction:

$$
\frac{\partial}{\partial Y}(\ldots) \equiv 0
$$

The model then becomes 2(1/2)-dimensional in the sense that dynamics takes place in the $(X, Z)$-plane, but velocity normal to this plane is allowed. The equations then have an additional invariant: the geostrophic momentum

$$
M=v+f X .
$$

Using $M$, the expression for the potential vorticity is

$$
q=\left(f+\frac{\partial u}{\partial X}\right) \frac{\partial \theta}{\partial Z}-\frac{\partial u}{\partial Z} \frac{\partial \theta}{\partial X}=\frac{\partial(M, \theta)}{\partial(X, Z)}
$$

Note that the thermal wind balance can be rewritten in the form

$$
f \frac{\partial M}{\partial Z}=\frac{g}{\theta_{r}} \frac{\partial \theta}{\partial X}
$$

and, therefore, a potential $\Phi$ such that

$$
M=f^{-1} \frac{\partial \Phi}{\partial X}, \quad \theta=\frac{\theta_{r}}{g} \frac{\partial \Phi}{\partial Z}
$$

may be introduced for balanced states. In fact, $\Phi$ is geopotential "extended" by $f^{2}\left(X^{2} / 2\right): \Phi=\phi+f^{2}\left(X^{2} / 2\right)$.

The three invariants $(\theta, q$ and $M)$ and the potential $\Phi$ will play an important rôle below.

\subsection{Lagrangian formulation}

In describing relaxation of a stratified fluid toward a geostrophic state, the Lagrangian formulation of the equations, like in the shallow-water model (Zeitlin et al., 2003), turns out to be a powerful tool because it makes a full use of the Lagrangian invariants. All quantities being independent of $Y$, we can describe the fluid by following the positions of material lines of fluid particles parallel to the $Y$ axis in the $(X, Z)$ plane. These lines of particles always remain straight; we will simply call them particles. The independent variables will be the initial positions $x$ and $z$ (Lagrangian labels), and time $t$. The dependent variables, $X(x, z, t)$ and $Z(x, z, t)$, define the position at time $t$ of the particle initially at $(x, z)$. The time derivative of a Lagrangian quantity (by definition, a material derivative) will be denoted by a dot. The conservation of $\theta, M$ and $q$ takes a simple form in Lagrangian coordinates:

$$
\dot{\theta}=0, \quad \dot{M}=0, \quad \dot{q}=0 .
$$


The incompressibility constraint is

$$
\frac{\partial(X, Z)}{\partial(x, z)}=1 .
$$

This equality is used, along with the properties of the Jacobians, to transform the horizontal pressure gradient term in (1a), and the vertical pressure gradient in (1b). The following equations are thus obtained:

$$
\begin{aligned}
\ddot{X}+f^{2} X+\frac{\partial(\phi, Z)}{\partial(x, z)} & =f\left(v_{I}+f x\right)=f M_{I}, \\
\frac{\partial(X, \phi)}{\partial(x, z)} & =g \frac{\theta_{I}}{\theta_{r}},
\end{aligned}
$$

where $v_{I}(x, z), M_{I}(x, z)$ and $\theta_{I}(x, z)$ are initial distributions of the jet velocity, geostrophic momentum and potential temperature. Note that the initial conditions are explicitly included in the equations.

In the above equations the geopotential $\phi$ can be eliminated by cross-differentiation, yielding two nonlinear equations for $X$ and $Z$ :

$$
\begin{aligned}
\frac{\partial(X, \ddot{X})}{\partial(x, z)}-\frac{\partial\left(X, f M_{I}\right)}{\partial(x, z)}+\frac{g}{\theta_{r}} \frac{\partial\left(\theta_{I}, Z\right)}{\partial(x, z)} & =0 \\
\frac{\partial(X, Z)}{\partial(x, z)} & =1
\end{aligned}
$$

where only the term $\ddot{X}$ involves time derivatives. These two equations are the primitive equations in the Lagrangian form.

\section{Finding the adjusted state: the diagnostic geostrophic adjustment problem}

An essential advantage of the Lagrangian formulation for flows not varying in one horizontal direction arises while identifying the balanced (slow) and unbalanced (fast) components of the flow. For flows not varying in one horizontal direction, the geostrophic balance is an exact stationary solution of the equations. Hence, the balanced part of the flow is stationary, and the rest forms the unbalanced part. This separation is evident from equations (9a): the balanced part of the flow is a solution to the equations without the time derivatives. The balanced state is given by $(\bar{X}(x, z)$, $\bar{Z}(x, z))$, which satisfy

$$
\begin{aligned}
-\frac{\partial\left(\bar{X}, f M_{I}\right)}{\partial(x, z)}+\frac{g}{\theta_{r}} \frac{\partial\left(\theta_{I}, \bar{Z}\right)}{\partial(x, z)} & =0, \\
\frac{\partial(\bar{X}, \bar{Z})}{\partial(x, z)} & =1 .
\end{aligned}
$$


Note, that the first of equations (10) simply states the thermal wind balance rewritten in $(x, z)$ variables (cf. (5)); the second is the incompressibility condition. Equation (10b), along with the conservations of $M$ and $\theta$, implies that the PV (cf. (4)) is also conserved. Note also that the balanced state thus defined generally differs from what would be defined as the balanced state from an inversion of the Eulerian PV distribution (e.g. Hoskins et al., 1985).

We will analyse below this problem with two types of boundary conditions. We will always assume that the initial jet/front-like state is horizontally localized, meaning that fluid particles at $x \rightarrow \pm \infty$ are not displaced. We will consider then our flows either in a slab, requiring that vertical boundaries remain themselves:

$$
\bar{Z}(x, 0)=0, \quad \bar{Z}(x, H)=H,\left.\quad \bar{X}\right|_{x \rightarrow \pm \infty}=x,
$$

or on the whole $(x, z)$-plane by requiring that at $z \rightarrow \pm \infty$ the potential temperature profile tend to a given stable, let us say linear, stratification:

$$
\left.\theta\right|_{z \rightarrow \pm \infty}=\theta_{r} \frac{N^{2}}{g} z, \quad N=\text { const },\left.\quad \bar{X}\right|_{x \rightarrow \pm \infty}=x
$$

The first equation means that fluid particles at $z \rightarrow \pm \infty$ are not displaced: $\left.\bar{Z}\right|_{z \rightarrow \pm \infty}=z$. The equation for $X$ may be also rewritten as that for $M$, as both coincides (up to a constant) at infinity for localized velocity distributions.

If, starting from the initial state, the fluid reaches a stationary state in course of a dissipationless evolution, this state is necessarily a solution to the above equations. As in (Zeitlin et al., 2003), the problem of existence and uniqueness of solutions to equation (10) then arises. We will address it first for jet/front-like configurations in the unbounded domain.

\subsection{Criterion of existence and uniqueness of the adjusted state in the unbounded domain}

In the case of arbitrary distribution of PV it is more convenient to use the PV equation (4) which, together with $(6 a, b)$, gives

$$
\frac{\partial^{2} \Phi}{\partial X^{2}} \frac{\partial^{2} \Phi}{\partial Z^{2}}-\left(\frac{\partial^{2} \Phi}{\partial X \partial Z}\right)^{2}=\frac{g f}{\theta_{r}} q
$$

where $\mathrm{PV}$ in the right-hand side is understood as a function of $(X, Z)$. This is the Monge-Ampère equation and it is to be solved with boundary conditions (12). Although these are formally Neumann-type boundary conditions, it is easy to see that they mean that far enough from the origin the function $\Phi$ has the form

$$
\Phi_{|X|,|Z| \rightarrow \infty}=\frac{1}{2} f^{2} X^{2}+\frac{1}{2} N^{2} Z^{2}
$$

This means that on some distant ellipse (which is a convex curve) $\left(f^{2} X^{2}+N^{2} Z^{2}\right) / 2=$ constant, the function $\Phi$ is constant and, thus, the problem of finding the adjusted state 
is reduced to the first (Dirichlet) boundary-value problem for the Monge-Ampere equation. Existence of solution is guaranteed if the right hand side, i.e. PV, is continuous and positive (cf. Pogorelov, 1973; Ch. 8, Sect. 3, Theorem 2a). Moreover, if a condition of convexity is added on $\Phi$, which is the case of (14), the solution is unique (Pogorelov, 1973, Ch. 8, Sect. 4, Theorem 1). (There is always a trivial convex/concave degeneracy for Monge-Ampere equation: think of this equation with right hand side equal to 1 with the boundary condition 1 on the unit circle: both $\left(X^{2}+Z^{2}\right) / 2$ and its opposite are solutions, but only the first one is convex).

Thus, for positive PV, which is a condition of absence of symmetric instability (cf. e.g. Bennetts and Hoskins, 1979; Cho et al., 1993), the adjusted state exists and is unique in the absence of boundaries. Note that this criterion is exactly the same as for fronts in rotating shallow water (Zeitlin et al., 2003). Therefore the major question of existence and uniqueness of the adjusted state may be definitely answered in this case. We will see below in section 4.4 that the attainability of this state is not always certain. Moreover, a similar analysis is much more complicated in the presence of boundaries.

\subsection{Positive $P V$ configurations in domains with vertical boundaries: obstacles for the existence proof}

Motivated by the previous result, let us consider below a fluid with arbitrary, strictly positive PV in the vertically bounded domain. As boundary conditions in this case are to be imposed on $X$ and, especially, $Z$ themselves, cf. (11), it is more convenient to use $M$ and $\theta$ as independent variables in the Monge-Ampère equation. For strictly positive $\mathrm{PV}$ this change of variables is well-defined because the Jacobian of the transformation is equal to the PV (cf. (4)).

In $(M, \theta)$ coordinates, the thermal wind relation takes the form

$$
f \frac{\partial X}{\partial \theta}=\frac{g}{\theta_{r}} \frac{\partial Z}{\partial M}
$$

Hence a "potential" $\Psi$ for the final positions of the fluid particles may be introduced:

$$
X=\frac{g}{\theta_{r}} \frac{\partial \Psi}{\partial M}, \quad Z=f \frac{\partial \Psi}{\partial \theta} .
$$

The Jacobian of the transformation from $(x, z)$ to $(X, Z)$ can be rewritten as

$$
\frac{\partial(X, Z)}{\partial(M, \theta)} \frac{\partial(M, \theta)}{\partial(x, z)}=1
$$

from which we can obtain, replacing $X$ and $Z$ by their expressions (16 a,b) the following Monge-Ampère equation for $\Psi$ :

$$
\frac{\partial^{2} \Psi}{\partial M^{2}} \frac{\partial^{2} \Psi}{\partial \theta^{2}}-\left(\frac{\partial^{2} \Psi}{\partial M \partial \theta}\right)^{2}=\frac{\theta_{r}}{g f} \frac{1}{q}
$$


Assuming once again that fluid on the boundaries remains there (cf. (11)), this equation has oblique Neumann-type boundary conditions

$$
\begin{gathered}
\frac{\partial \Psi}{\partial \theta}\left(M_{ \pm}(s), \theta_{ \pm}(s)\right)=\frac{z_{ \pm}}{f}, \\
\left(\frac{g}{\theta_{r}} \frac{\partial \Psi}{\partial M}, f \frac{\partial \Psi}{\partial \theta}\right) \rightarrow(x(M, \theta), z(M, \theta)) \quad \text { as } M \rightarrow \pm \infty,
\end{gathered}
$$

where $\left(M_{ \pm}(s), \theta_{ \pm}(s)\right)$ define the upper $\left(z_{+}=H\right)$ and lower boundaries $\left(z_{-}=0\right)$ in the $(M, \theta)$ space, $s$ is a coordinate along those boundaries, $x$ and $z$ are initial positions.

Known mathematical results on elliptic Monge-Ampère with Neumann boundary conditions (Lions et al., 1986; Urbas, 1998) in a convex domain are, unfortunately, not applicable here because, first, our equation does not have the standard Neumann boundary conditions, and second, the domain in $(M, \theta)$ space is generally not convex. Hence, the main obstacle to establish existence and uniqueness theorem for the general geostrophic adjustment problem in stratified fluid comes from the presence of boundaries. This is not surprising, because in all studies of frontogenesis, which is a tightly related problem, see below, the singularity appears at the boundaries.

It is worth mentioning at this point that much research has been carried out on the Monge-Ampère equation in the bounded domains in the context of the semigeostrophic approximation, especially by Cullen and collaborators (Cullen, 1983; Cullen and Purser, 1984; Cullen and Purser, 1989; Cullen et al., 1991) in view of extending the semi-geostrophic description of frontogenesis beyond the point where a discontinuity forms. The semi-geostrophic description of the flow involves solution of an elliptic Monge-Ampère equation at each timestep. Positiveness of the PV was shown to be essential for stability (Shutts and Cullen, 1987) and is a necessary condition for solving the equation. It was shown (Cullen and Purser, 1984; Cullen and Purser, 1989; Cullen et al., 1991) that it was always possible to rearrange fluid elements, respecting conservation of potential temperature and the Lagrangian evolution of geostrophic momentum, in order to obtain a solution. However, these solutions, as a rule, include discontinuities in $v$ and $\theta$, and the question whether they are reached in course of evolution remains open.

The fact that equation (18) appears in the context of the semi-geostrophic frontogenesis is an indication that the two problems are related. This relation is rendered mathematically precise in Appendix A. Advancing in time in the semi-geostrophic description of frontogenesis is equivalent to considering a sequence of adjusted states corresponding to initial states with tighter and tighter potential temperature gradients in the horizontal, the squeezing of the potential temperature gradients being provided by advection by the external deformation velocity field. Thus, the deformation field provides a parameter allowing to pass from "normal" to "catastrophic" geostrophic adjustment. Semi-geostrophic approximation in our 2(1/2)-dimensional context means exactly filtering the fast component and, thus, remaining on the diagnostic level, time-changes appearing exclusively due to parametric dependence on the deformation field. Thus the mathematics of the deformation and spontaneous frontogenesis are exactly the same. From the numerical studies of the semi-geostrophic frontogenesis it is known that discontinuities form for fluids with arbitrary distributions of positive PV. Hence, we expect that for initial states that have strong enough 
horizontal variations in the potential temperature, a smooth adjusted state does not exist.

Below we illustrate the non-existence of the adjusted state in the bounded domain for strong enough gradients of the initial fields on the examples of zero- and uniform PV distributions, where Lagrangian description allows to treat the problem analytically. As expected, singularities form at the boundaries.

\subsection{The zero-PV case}

We illustrate the non-existence of smooth adjusted states by constructing solutions for a zero-PV (ZPV) fluid in an horizontally infinite slab with arbitrary initial conditions. The advantage of the ZPV case is that its simplicity makes it possible to obtain analytically the necessary and sufficient condition for the existence of an adjusted state from an initial state having a horizontal temperature gradient and a jet. The case of a ZPV fluid was introduced as the technically simplest one in studies of frontogenesis (Hoskins and Bretherton, 1972). The initial conditions are

$$
\theta_{I}=\theta_{I}(x) \text { and } \quad v_{I}=v_{I}(x)
$$

hence $M_{I}=M_{I}(x)=v_{I}(x)+f x$. After substitution in (10a), this yields

$$
\frac{\partial \bar{X}}{\partial z} f M_{I}^{\prime}+\frac{\partial \bar{Z}}{\partial z} \frac{g \theta_{I}^{\prime}}{\theta_{r}}=0
$$

where the prime denotes the $x$-derivatives. For compactness we introduce the following quantity: $\Theta_{I}^{\prime}=g \theta_{I}^{\prime} / \theta_{r}$. Equation (21) can be integrated to give:

$$
\bar{X}=\frac{\mathcal{F}(x)}{f M_{I}^{\prime}}-\frac{\Theta_{I}^{\prime}}{f M_{I}^{\prime}} \bar{Z}
$$

where $\mathcal{F}(x)$ is an arbitrary function. As $M_{I}$ and $\theta_{I}$ depend only on $x$, (22) shows that isentropes (or isolines of geostrophic momentum) are straight lines in the final state.

Expression (22) can then be substituted into (10b), yielding an equation for $Z$ that can be straightforwardly integrated:

$$
\bar{Z}^{2}\left(\frac{\Theta_{I}^{\prime}}{f M_{I}^{\prime}}\right)^{\prime}-2\left(\frac{\mathcal{F}}{f M_{I}^{\prime}}\right)^{\prime} \bar{Z}+2(\mathcal{G}(x)+z)=0 .
$$

Here $\mathcal{G}(x)$ is a second unknown function of $x$. The two functions $\mathcal{F}(x)$ and $\mathcal{G}(x)$ can be determined by using the boundary condition $X \rightarrow x$, for $x \rightarrow \pm \infty$ and by requiring that fluid parcels initially on the upper and lower boundaries remain there:

$$
\bar{Z}(x, 0)=0 \quad \text { and } \quad \bar{Z}(x, H)=H .
$$


The following expressions are thus obtained:

$$
\begin{aligned}
& \bar{X}=x+\mathcal{A}(x)\left(\frac{H}{2}-\bar{Z}\right) \\
& \bar{Z}=\frac{1}{\mathcal{A}^{\prime}(x)}\left[1+\frac{H}{2} \mathcal{A}^{\prime}(x)-\sqrt{\left(1+\frac{H}{2} \mathcal{A}^{\prime}(x)\right)^{2}-2 z \mathcal{A}^{\prime}(x)}\right]
\end{aligned}
$$

where notation $\mathcal{A}=\Theta_{I}^{\prime} / f M_{I}^{\prime}$ is introduced for compactness.

If a discontinuity forms, this happens on a boundary, where $\partial X / \partial x(x, 0)=0$ or $\partial X / \partial x(x, H)=0$. From (25a), this will happen if

$$
\frac{\partial \bar{X}}{\partial x}(x, 0)=1+\frac{1}{2} H \mathcal{A}^{\prime}(x)=0, \quad \text { or } \quad \frac{\partial \bar{X}}{\partial x}(x, H)=1-\frac{1}{2} H \mathcal{A}^{\prime}(x)=0,
$$

or, coming back to the original notation:

$$
\frac{g}{f \theta_{r}}\left(\frac{\theta_{I}^{\prime}}{f+v_{I}^{\prime}}\right)^{\prime}=\frac{g}{f \theta_{r}}\left(\frac{\theta_{I}^{\prime \prime}}{f+v_{I}^{\prime}}-\frac{\theta_{I}^{\prime} v_{I}^{\prime \prime}}{\left(f+v_{I}^{\prime}\right)^{2}}\right)= \pm \frac{2}{H}
$$

This condition was first found in (Blumen and $\mathrm{Wu}, 1995)$. The presence of the initial velocity shear $v_{I}$ thus means that the isentropes in initially anticyclonic regions $v_{I}^{\prime}<0$ will have shallower slopes than in the absence of initial velocity (cf. (25a)). The anticyclonic relative vortictiy of $v_{I}$ acts to locally lower down the Coriolis force, hence allowing the fluid to spread more. Conversely, in initially cyclonic regions, the shear enhances locally the Coriolis force and the fluid spreads less, yielding steeper slopes of the isentropes. As a consequence, the regions of initially anticyclonic shear will favor appearance of the discontinuity, either on the ground or on the top.

This situation is illustrated by figures 1 and 2 . Note that the inversion of (25a) giving $x=x(\bar{X}, \bar{Z})$ is sufficient to find the profiles of $\theta$ and $v$ in the adjusted state. As $\theta$ is a Lagrangian invariant the change of variables $\theta_{I}(x) \rightarrow \theta(x(\bar{X}, \bar{Z}))$ is enough. The profile of $v$ is obtained from the Lagrangian invariance of the geostrophic momentum $M$ :

$$
v(\bar{X}, \bar{Z})=f(x(\bar{X}, \bar{Z})-\bar{X})+v_{I}(x(\bar{X}, \bar{Z}))
$$

In the case presented at figures 1 and 2 the inversion was made numerically. It is worth noting that for $\theta_{I} \propto \arctan (x)$ and zero $v_{I}$ all calculations may be done analytically using Cardano formulas for solving (25a).

Although the ZPV case is special, it gives indications as to the effect of initial horizontal shear impossible to obtain analytically in more realistic situations. In particular, it is important to stress that the regions having initially anticyclonic shear favor formation of a singularity, although the discontinuity will be associated, as in the frontogenesis process (see Hoskins and Bretherton (1972) and appendix A) with divergent cyclonic relative vorticity, see figure $2 \mathrm{~d}$. Thus, Lagrangian approach allows for a full clarification of this point which was raised but not answered in the preceding work (Wu and Blumen, 1995). 
(a)

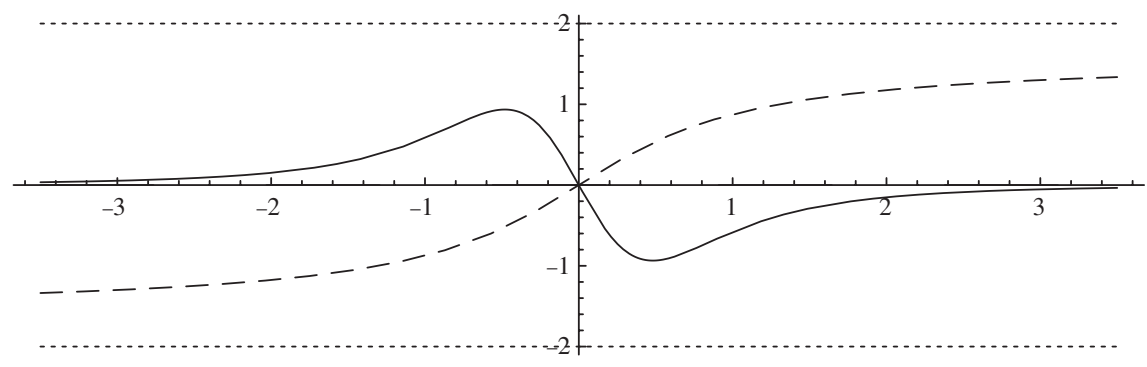

(b)

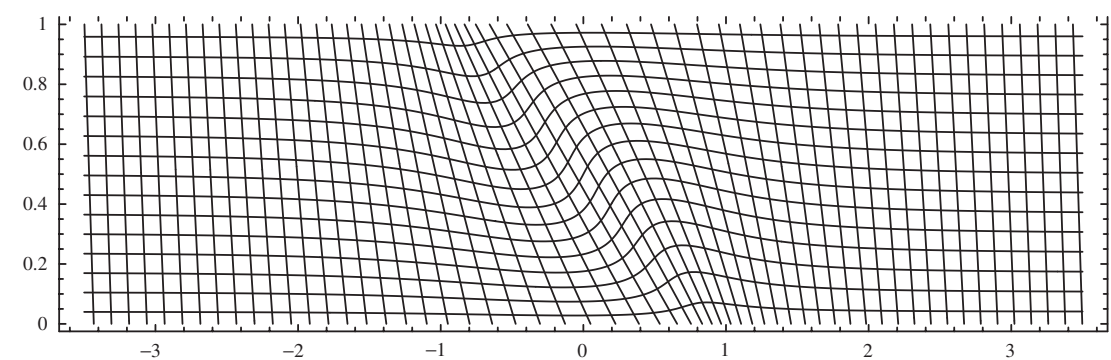

(c)



(d)

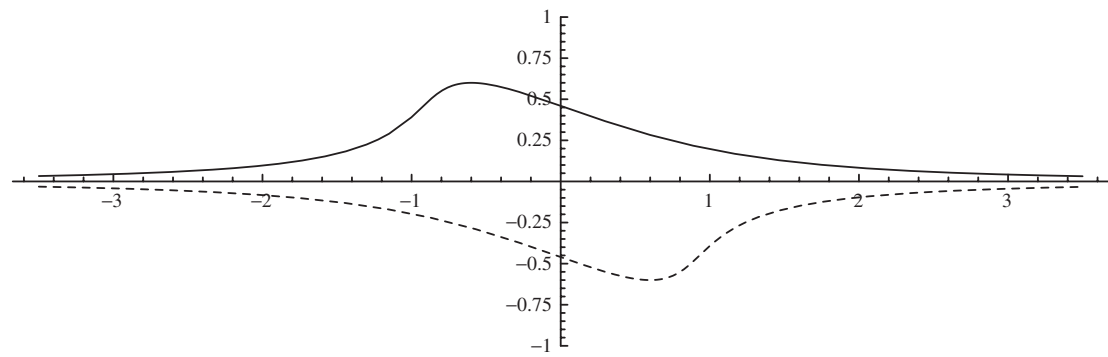

Figure 1. Geostrophically adjusted state for an initial state with zero potential vorticity and $v_{I}(x)=0$. Panel (a) shows the profiles of $\theta_{I}=\arctan (1.2 x)$ (dashed; defined up to an additive constant), and $\mathcal{A}^{\prime}$ (plain). All constants being taken equal to 1 , the threshold for the formation of a discontinuity is $\mathcal{A}^{\prime}= \pm 2$; indicated in a) by short-dashed lines. (b) final positions of particles initially forming a regular grid; (c) isotachs of the final velocity field; (d) the velocity on top (plain) and bottom boundaries (dashed) as a function of $X$. 
(a)

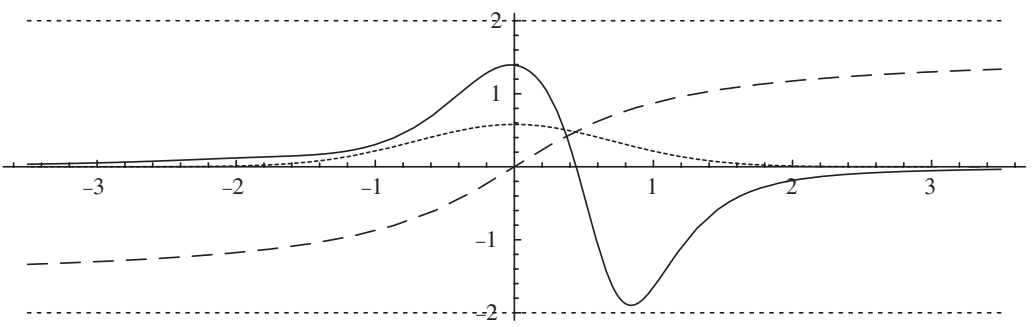

(b)

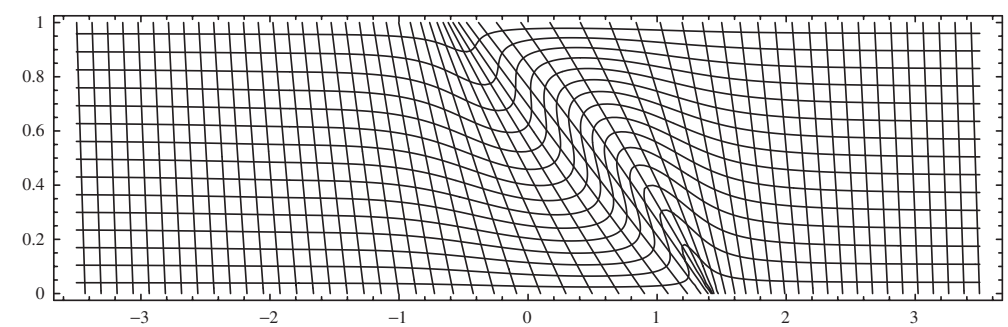

(c)

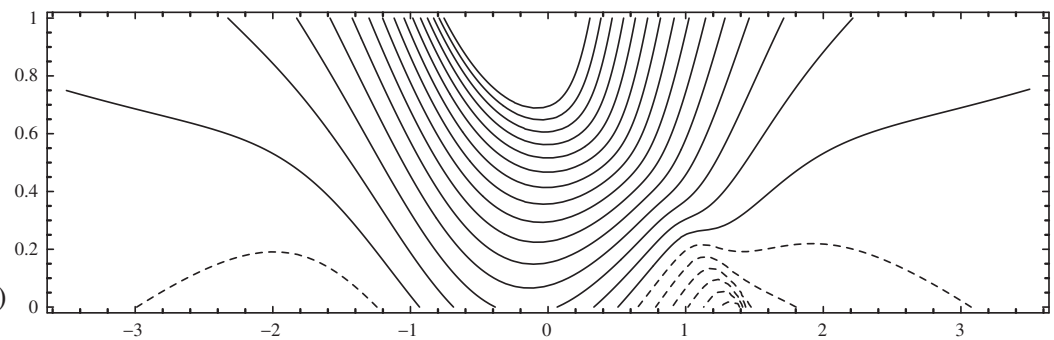

(d)

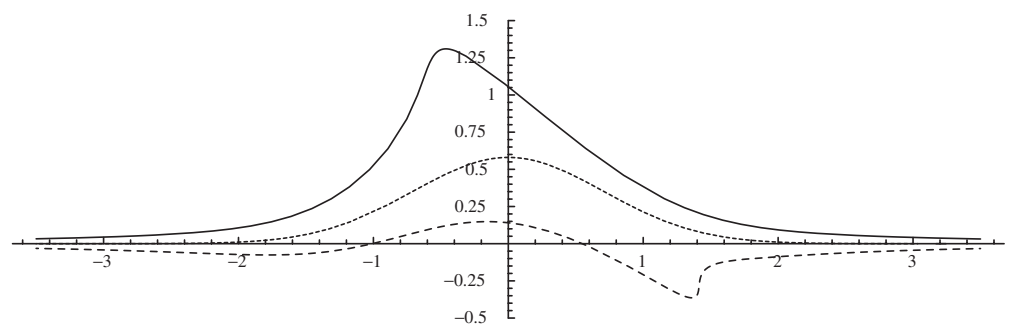

Figure 2. Same as figure 1, but with an initial condition including a non-zero velocity distribution $v_{I}(x)=0.58 \exp \left(-x^{2}\right)$, shown in (a) as the dotted line. The region of initial anticyclonic shear $(x>0)$ corresponds to the region where $\theta_{I}^{\prime \prime}$ is negative, and hence favors the formation of a discontinuity near the ground, as can be seen in (c) and (d). Note that the maximum positive value of $\mathcal{A}^{\prime}$ has been shifted to the region of initial anticyclonic shear.

We can estimate $\mathcal{A}$ for temperature gradients typically observed in the atmosphere ${ }^{1}$. Taking $H$ to be $5 \mathrm{~km}$, we find that the threshold for non-existence of the adjusted state is $\sim 4 \times 10^{-4} \mathrm{~m}^{-1}$. Considering a situation without shear with an unbalanced potential temperature gradient of $2 \mathrm{~K}$ per $100 \mathrm{~km}$ and the same typical scale of variation

\footnotetext{
${ }^{1}$ Unbalanced 'initial' conditions can be forced, for example, by horizontal gradients in heating at land-sea boundaries, or between a clear and a cloudy area (see Blumen and Williams, 2001 and refrences therein).
} 
$(100 \mathrm{~km})$, we find that $\mathcal{A}^{\prime}$ is of order $6 \times 10^{-4} \mathrm{~m}^{-1}$. This gives an indication that the threshold may be routinely reached by atmospheric mesoscale motions.

The study of the ZPV case thus shows that a smooth adjusted state may not exist for initial states of the vertically bounded fluid with strong enough horizontal gradients of potential temperature and/or strong enough anticyclonic shear. The question remains open as to solutions with a discontinuity of the along-jet velocity in the interior of the fluid layer. Such solutions were constructed in (Kalashnik, 1998, 2000), but the evolution of the fluid toward such a state may be prevented by turbulent processes.

Another important question is that of attainability of the adjusted state constructed above: indeed it is impossible for inertia-gravity waves (IGW) to propagate away from a (localized) density anomaly as there is no background stratification. This casts doubts, in the ZPV fluid, on the evacuation of excess energy by IGW radiation which is the basic ingredient of the standard adjustment scenario as discussed in Introduction (cf. also Blumen and Williams, 2001).

\subsection{The uniform $P V$ case}

Geostrophic adjustment of a fluid with uniform PV (UPV), another technically simplified case, was studied by Blumen and Wu (1995) and Kalashnik (1998). Using geostrophic momentum $M$ and $\bar{Z}$ as coordinates (cf. Hoskins and Bretherton, 1972), they obtained a Laplace equation for the potential temperature. Below, using Lagrangian variables, we show that finding positions of particles in the adjusted state is equivalent to solving the Laplace equation with Neumann boundary conditions. As an initial state of uniform nonzero PV, we take

$$
v_{I}=0, \quad \text { and } \quad \theta_{I}=\theta_{r}\left(\frac{N^{2}}{g} z+\psi(x)\right),
$$

where $\psi(x)$ is an arbitrary function with a compact support derivative, and $N^{2}$ is constant. After substitution into (9a), this yields the following equations:

$$
\begin{aligned}
-\frac{\partial\left(\bar{X}, f^{2} x\right)}{\partial(x, z)}+g\left(\psi^{\prime} \frac{\partial \bar{Z}}{\partial z}-\frac{N^{2}}{g} \frac{\partial \bar{Z}}{\partial x}\right) & =0 \\
\frac{\partial(\bar{X}, \bar{Z})}{\partial(x, z)} & =1 .
\end{aligned}
$$

We change variables, and use the final vertical position $\bar{Z}$ of a fluid particle instead of $z$. Hence the dependent variables are $\bar{X}(x, \bar{Z})$ and $z(x, \bar{Z})$, and the Jacobian of the transformation is

$$
\frac{\partial(x, \bar{Z})}{\partial(x, z)}=\frac{\partial \bar{Z}}{\partial z}
$$


In this new system of coordinates, equations (30a) and (30b) become

$$
\begin{aligned}
-\frac{\partial\left(\bar{X}, f^{2} x\right)}{\partial(x, \bar{Z})}+g \psi^{\prime}+N^{2} \frac{\partial z}{\partial x} & =0, \\
\frac{\partial \bar{X}}{\partial x}-\frac{\partial z}{\partial \bar{Z}} & =0 .
\end{aligned}
$$

Combining them to eliminate $z$, we obtain a Laplace equation which describes the adjusted state

$$
f^{2} \frac{\partial^{2} \bar{X}}{\partial \bar{Z}^{2}}+N^{2} \frac{\partial^{2} \bar{X}}{\partial x^{2}}=0
$$

The boundary conditions follow from (11) and (32a):

$$
\frac{\partial \bar{X}}{\partial \bar{Z}}=\frac{g}{f^{2}} \psi^{\prime}, \quad \text { for } \bar{Z}=0, H
$$

By a proper rescaling of $x, \bar{Z}$ and $\psi$ we get a standard Neumann problem for $\bar{X}$ obeying the Laplace equation in a unit strip $-\infty<x<+\infty, 0<\bar{Z}<1$ with the normal derivative on both boundaries equal to $\psi^{\prime}$ and $\bar{X} \rightarrow x$ as $x \rightarrow \pm \infty$. As is well known (see e.g. Laurentiev and Shabat 1987), a solution of the Neumann problem may be obtained from a solution $F(x, \bar{Z})=a(x, \bar{Z})+\mathrm{i} b(x, \bar{Z})$ of the corresponding Dirichlet problem for the conjugate analytic function. The boundary condition for the Dirichlet problem is obtained by integrating the Neumann boundary condition along the boundary. The Schwarz integral for the unit strip in the complex plane $\zeta=x+\mathrm{i} \bar{Z}$ which provides solution of the Dirichlet problem with boundary conditions $\left.a\right|_{\bar{Z}=0,1}=a_{0,1}$ is

$$
F(\zeta)=-\frac{\mathrm{i}}{2} \int_{-\infty}^{+\infty} \mathrm{d} t a_{0}(t) \operatorname{coth} \frac{\pi(t-\zeta)}{2}+\frac{\mathrm{i}}{2} \int_{-\infty}^{+\infty} \mathrm{d} t a_{1}(t) \tanh \frac{\pi(t-\zeta)}{2}
$$

In our case $a_{0}=a_{1}=\psi$ and, after elementary transformations we get

$$
F(\zeta)=-\mathrm{i} \int_{-\infty}^{+\infty} \mathrm{d} t \frac{\psi(t)}{\sinh \pi(t-\zeta)}
$$

Separating the real part of the function $F$ we get

$$
a(x, \bar{Z})=\int_{-\infty}^{+\infty} \mathrm{d} t \psi(t) \frac{\cosh \pi(t-x) \sin \pi \bar{Z}}{\sinh ^{2} \pi(t-x)+\sin ^{2} \pi \bar{Z}}
$$

The corresponding solution of the Neumann problem for $\bar{X}$ is then obtained by the integration of the Cauchy-Riemann relation $\partial \bar{X} / \partial x=-\partial a / \partial \bar{Z}$ and taking into account 
the boundary conditions at infinity (cf. Kalashnik, 1998):

$$
\bar{X}(x, \bar{Z})=x+\int_{x}^{+\infty} \mathrm{d} t\left(1+\frac{\partial a}{\partial \bar{Z}}\right) .
$$

Thus, solution for $\bar{X}$ is available but it does not always correspond to a physical one. For $\bar{X}$ to describe a physical solution, the Jacobian (31) has to be nonzero everywhere, implying that (cf. (32b))

$$
\frac{\partial \bar{X}}{\partial x}>0
$$

everywhere. As can be easily seen from (38), (37), and demonstrated by previous studies (Blumen and $\mathrm{Wu}, 1995$; Kalashnik, 1998, 2000), for a given profile of $\psi$ there is a critical intensity of the initial $\theta$-gradient for which a discontinuity appears at the boundary. For initial gradients stronger than that critical value, a well-defined, continuous adjusted state no longer exists.

Thus, in both the ZPV and UPV cases in the vertically bounded domains, it is possible to explicitly construct solutions to equations (10) and (11) and we answer, by direct computation, the question of existence and uniqueness of the adjusted state. The adjusted state does not exist for intense enough initial gradients of potential temperature or moderate potential temperature gradients combined with intense enough shears. Whether this means that a corresponding discontinuous state is reached or that wave-breaking events deviate the relaxation process toward some other final state remains an open question. The nonexistence of an adjusted state even for positive constant PV in the presence of boundaries shows their importance in the adjustment process. We will see below that their role is also crucial in the relaxation toward an adjusted state when the unbalanced component of motion is not filtered.

\section{Perturbative description of the adjustment process}

We here consider, in the case when an adjusted state exists, the attainability of this state.

\subsection{Dynamics of small perturbations on the background of the adjusted state}

We start from the Lagrangian primitive equations $(9 \mathrm{a}, \mathrm{b})$ and assume that the initial conditions are such that an adjusted state $(\bar{X}, \bar{Z})$ exists. It is natural to introduce the deviations of the particle positions from equilibrium:

$$
X=\bar{X}+\chi, \quad Z=\bar{Z}+\zeta
$$

so that equation (9a) becomes

$$
\frac{\partial(\bar{X}+\chi, \ddot{\chi})}{\partial(x, z)}-\frac{\partial\left(\bar{X}+\chi, f M_{I}\right)}{\partial(x, z)}+\frac{g}{\theta_{r}} \frac{\partial\left(\theta_{I}, \bar{Z}+\zeta\right)}{\partial(x, z)}=0 .
$$


It is more convenient to use as independent variables the positions of the particles in the adjusted state $(\bar{X}, \bar{Z})$, rather than the initial positions $(x, z)$. When this change of variables is made in (40), two terms which express the thermal wind relation in the adjusted state cancel out. Furthermore, it is advantageous to express the gradients of $\bar{v}$ and $\theta$ in the adjusted state through the geopotential $\bar{\phi}$, making use of geostrophic and hydrostatic balances, respectively. Equation (40) then becomes

$$
\left(\frac{\partial^{2}}{\partial t^{2}}+f^{2}+\frac{\partial^{2} \bar{\phi}}{\partial \bar{X}^{2}}\right) \frac{\partial \chi}{\partial \bar{Z}}+\frac{\partial^{2} \bar{\phi}}{\partial \bar{X} \partial \bar{Z}}\left(-\frac{\partial \chi}{\partial \bar{X}}+\frac{\partial \zeta}{\partial \bar{Z}}\right) \frac{\partial^{2} \bar{\phi}}{\partial \bar{Z}^{2}} \frac{\partial \zeta}{\partial \bar{X}}=0
$$

The incompressibility condition (9a) gives

$$
\frac{\partial \chi}{\partial \bar{X}}+\frac{\partial \zeta}{\partial \bar{Z}}+\frac{\partial(\chi, \zeta)}{\partial(\bar{X}, \bar{Z})}=0
$$

In the previous sections we did not introduce the characteristic scales in the equations in order to emphasize that the analysis did not depend on scaling assumptions. We will now introduce the scales appropriate for the perturbative description.

As we are interested in inertia-gravity waves, time will be scaled by $f^{-1}$. The horizontal displacements scale is $U / f$, where $U$ is the order of magnitude of the transverse velocity perturbations. These are small with respect to the jet velocity, of order $V$, so we can write $U=\delta V$, with $\delta \ll 1$. This scaling applies to flows in which the velocity field is dominated by a jet in one direction. The typical horizontal and vertical lengthscales are $L$ and $H$, respectively. The scale of the vertical displacements is $U H / f L$, from the continuity equation. We thus obtain

$$
\begin{array}{r}
\left(\frac{\partial^{2}}{\partial t^{2}}+1+R o \frac{\partial^{2} \bar{\phi}}{\partial \bar{X}^{2}}\right) \frac{\partial \chi}{\partial \bar{Z}}+R o \frac{\partial^{2} \bar{\phi}}{\partial \bar{X} \partial \bar{Z}}\left(-\frac{\partial \chi}{\partial \bar{X}}+\frac{\partial \zeta}{\partial \bar{Z}}\right)-B u \frac{\partial^{2} \bar{\phi}}{\partial \bar{Z}^{2}} \frac{\partial \zeta}{\partial \bar{X}}=0 \\
\frac{\partial \chi}{\partial \bar{X}}+\frac{\partial \zeta}{\partial \bar{Z}}+\delta R o \frac{\partial(\chi, \zeta)}{\partial(\bar{X}, \bar{Z})}=0
\end{array}
$$

where $R o=V / f L$ is the Rossby number and $B u=N^{2} H^{2} / f^{2} L^{2}$ is the Burger number. As we consider intense jets, the Rossby number is of order 1 . The Burger number will also be taken of order 1 . The small parameter is $\delta$; hence we expand $\chi$ in asymptotic series

$$
\chi=\chi^{(0)}+\delta \chi^{(1)}+\cdots
$$

and similarly for $\zeta$. Then from (43b) we obtain at leading order

$$
\frac{\partial \chi^{(0)}}{\partial \bar{X}}+\frac{\partial \zeta^{(0)}}{\partial \bar{Z}}=0
$$


and hence there exists a streamfunction $\psi^{(0)}$ such that

$$
\chi^{(0)}=-\frac{\partial \psi^{(0)}}{\partial \bar{Z}}, \quad \zeta^{(0)}=\frac{\partial \psi^{(0)}}{\partial \bar{X}} .
$$

Equation (43a) can then be rewritten

$$
\left(\frac{\partial^{2}}{\partial t^{2}}+1+\frac{\partial^{2} \bar{\phi}}{\partial \bar{X}^{2}}\right) \frac{\partial^{2} \psi^{(0)}}{\partial \bar{Z}^{2}}-\frac{\partial^{2} \bar{\phi}}{\partial \bar{X} \partial \bar{Z}} \frac{\partial^{2} \psi^{(0)}}{\partial \bar{X} \partial \bar{Z}}+\frac{\partial^{2} \bar{\phi}}{\partial \bar{Z}^{2}} \frac{\partial^{2} \psi^{(0)}}{\partial \bar{X}^{2}}=0 .
$$

The boundary conditions are provided by the requirements of no vertical displacement for parcels on the top and bottom boundaries, and of bounded displacements. The first condition implies that $\psi^{(0)}$ is constant on the top and bottom boundaries (cf. the second expression in (46)). As we suppose that there is no overall displacement of the fluid layer in the $X$-direction, these constants are equal; furthermore, as they are arbitrary they can be set equal to zero, yielding: $\psi^{(0)}(\bar{X}, 0)=\psi^{(0)}(\bar{X}, 1)=0$. The second condition, combined with the first one, imposes that $\psi^{(0)}$ remains bounded as $\bar{X} \rightarrow \pm \infty$ (see the first expression in (46)). Equation (47) closely resembles the homogeneous part of the Sawyer-Eliassen equation (see e.g. Holton, 1992) except for the addition of the term with the double-time derivative, which makes (47) a prognostic equation. In the Sawyer-Eliassen equation, this term is absent because the fast time has been filtered out by particular scaling, making the equation diagnostic.

Analytical progress is difficult because of spatially varying coefficients in this equation. Some insight can nevertheless be gained by considering the Fouriertransformation of $\psi^{(0)}$ in time. The equation for the spatial structure of the Fouriercomponent of frequency $\omega,\left(\psi_{\omega}^{(0)}(\bar{X}, \bar{Z})\right)$ is

$$
\left(1+\frac{\partial^{2} \bar{\phi}}{\partial \bar{X}^{2}}-\omega^{2}\right) \frac{\partial^{2} \psi_{\omega}^{(0)}}{\partial \bar{Z}^{2}}-2 \frac{\partial^{2} \psi_{\omega}^{(0)}}{\partial \bar{X} \partial \bar{Z}} \frac{\partial^{2} \bar{\phi}}{\partial \bar{X} \partial \bar{Z}}+\frac{\partial^{2} \psi_{\omega}^{(0)}}{\partial \bar{X}^{2}} \frac{\partial^{2} \bar{\phi}}{\partial \bar{Z}^{2}}=0
$$

This equation is hyperbolic in the variables $\bar{X}$ and $\bar{Z}$ when

$$
\left(1+\frac{\partial^{2} \bar{\phi}}{\partial \bar{X}^{2}}-\omega^{2}\right) \frac{\partial^{2} \bar{\phi}}{\partial \bar{Z}^{2}} \phi-\left(\frac{\partial^{2} \bar{\phi}}{\partial \bar{X} \partial \bar{Z}}\right)^{2}=q-\frac{\partial^{2} \bar{\phi}}{\partial \bar{Z}^{2}} \omega^{2}<0,
$$

and elliptic otherwise. Given the boundary conditions, hyperbolicity of this equation in some region is a necessary condition for a nontrivial solution, i.e. for waves at frequency $\omega$, to exist.

Away from the jet, $q \sim \partial^{2} \bar{\phi} / \partial \bar{Z}^{2}$ and the equation is hyperbolic for all $\omega>1$ if the condition of static stability $\partial^{2} \bar{\phi} / \partial \bar{Z}^{2}>0$ is satisfied. Equation (48) becomes parabolic for large $X$ in a zero-PV fluid with no stratification $\left(\partial^{2} \bar{\phi} / \partial \bar{Z}^{2}=0\right)$. For $\omega<1$, equation (48) may be hyperbolic locally in regions of anticyclonic shear. The modes with $\omega<1$, if they exist, are evanescent outside of the anticyclonic region of the jet; hence they are trapped. Qualitatively (Klein and Tréguier, 1995), the trapping of waves can be understood in the following way: the relative vorticity of the jet modifies locally the 
Coriolis parameter to the value $1+\partial \bar{v} / \partial \bar{X}$, making it possible to have waves with subinertial frequency in the anticyclonic region (Kunze, 1985).

Once potential temperature and velocity profiles of the adjusted state are known, equation (48) in hyperbolic regions may be solved by the method of characteristics. However, such study is rather involved in the general baroclinic case and lies beyond the scope of the present paper. In the following section a simplifying assumption of barotropic jet will be made to allow further (semi-)analytical progress. (Note that the presence of the time-derivative in (47) makes the change of variables applied in the standard analysis of the Sawyer-Eliassen equation ineffective.)

\subsection{Trapped waves in a barotropic jet}

The non-dimensional geopotential describing a balanced barotropic jet is

$$
\bar{\phi}=\Phi(\bar{X})+\frac{1}{2} \bar{Z}^{2}
$$

The mean stratification, which we suppose constant, is given by the second derivative of the second term in this expression and the jet velocity is $\bar{v}=\Phi^{\prime}$, where the prime denotes the $X$-derivative $\Phi^{\prime}=\partial \Phi / \partial \bar{X}$. In order to provide illustrations, an example $\Phi$ which allows for explicit analytical solution is considered below and in Appendix B. It consists in a compact jet with piecewise-linear relative vorticity which is shown in figure 3. This example is useful in providing illustrations and analytical solutions. The results obtained below are general and do not restrict to this example.

If an unbalanced fast component is added to (50), the equation for its evolution is (cf. (47)):

$$
\left(1+\Phi^{\prime \prime}+\frac{\partial^{2}}{\partial t^{2}}\right) \frac{\partial^{2} \psi^{(0)}}{\partial \bar{Z}^{2}}+\frac{\partial^{2} \psi^{(0)}}{\partial \bar{X}^{2}}=0
$$

It allows a separation of variables and a regular Sturm-Liouville problem in the vertical results; given the boundary conditions, the vertical eigenfunctions $\operatorname{are} \sin (n \pi \bar{Z})$. Hence the solutions is

$$
\psi^{(0)}(\bar{X}, \bar{Z}, t)=\sum_{n} \sin (n \pi \bar{Z}) \psi_{n}^{(0)}(\bar{X}, t)
$$

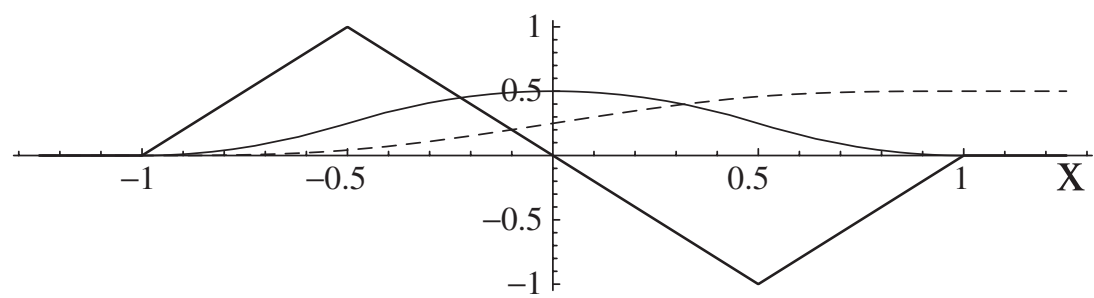

Figure 3. Profiles used to obtain explicit analytical solutions: $\Phi$ (dashed), jet velocity $\Phi^{\prime}$ (plain) and relative vorticity $\Phi^{\prime \prime}$ (thick) in appendix B. The parameters are the half-width $L$ and the maximum value of the relative vorticity $B$; here both are taken equal to 1 . 
We look for $\psi_{n}^{(0)}(\bar{X}, t)$ with a time-dependence of the form $e^{-i \omega t}$ and get a SturmLiouville problem on $(-\infty,+\infty)$. We denote by $\hat{\psi}_{n \omega}(x)$ the horizontal eigenfunction with vertical wavenumber $n$ and frequency $\omega$. The equation for $\hat{\psi}_{n \omega}(\bar{X})$ has the form of Shrödinger equation for stationary states of a particle in a potential (e.g. Messiah, 1995):

$$
\frac{\partial^{2} \hat{\psi}_{n \omega}}{\partial \bar{X}^{2}}-n^{2} \pi^{2}\left(1+\Phi^{\prime \prime}-\omega^{2}\right) \hat{\psi}_{n \omega}=0
$$

The factor $n^{2} \pi^{2}$ may be removed by rescaling $X$ as $S=n \pi \bar{X}$. This gives the equation:

$$
\frac{\partial \hat{\psi}_{n \omega}}{\partial S^{2}}-\left(1+\Phi^{\prime \prime}(S / n \pi)-\omega^{2}\right) \hat{\psi}_{n \omega}=0
$$

where the "potential" is $\left(1+\Phi^{\prime \prime}(S / n \pi)\right)$ and the eigenvalues are $\omega^{2}$. For any given profile of $\Phi$, the depth of the potential is always the same, but its width depends on the vertical wavenumber $n$ : the smaller the vertical scale of the waves, the wider the potential.

It is known that Shrödinger equation (54) has a continuous and a discrete spectrum (e.g. Messiah, 1995) of eigenvalues $\omega^{2}$. In our case, the potential $\left(1+\Phi^{\prime \prime}\right)$ tends to one as $\bar{X} \rightarrow \infty$; hence, for a given $n$, we have

- a continuous spectrum of solutions with $\omega>1$. This part of the spectrum is doubly degenerate (two independent solutions for each eigenvalue $\omega$ ) and corresponds to leftward and rightward propagating waves.

- a discrete spectrum of solutions with subinertial frequencies:

$$
\sqrt{\operatorname{Min}\left(1+\Phi^{\prime \prime}\right)}<\omega<1
$$

This part of the spectrum is nondegenerate, and corresponds to localized solutions. These solutions are exponentially decaying outside the region where $\left(1+\Phi^{\prime \prime}-\right.$ $\left.\omega^{2}\right)<0$, and have oscillatory character inside that region: they are trapped in the anticyclonic part of the jet. For any given $n$, the fundamental mode (lowest possible $\omega$, cf. figures $4 \mathrm{a}$ and $\mathrm{b}$ ) has no nodes; the next mode, if it exists, has one node (e.g. figure $4 \mathrm{c})$ ), etc...

For jets of the form displayed in figure 3 a semi-analytic analysis of the discrete spectrum is possible. Analytic expressions for the trapped modes are obtained with the help of Airy functions (cf. appendix B). Examples are presented in figure 4. The corresponding dispersion relation $\omega(n)$ is shown in figure 5. For the fundamental mode, the frequency tends very slowly toward the lower bound as $n \rightarrow \infty$.

Trapped subinertial modes have been investigated in rectilinear jets with small Rossby numbers by Kunze (1985), for waves that have small horizontal scales relative to the width of the jet, using the WKB approximation. He showed that sub-inertial waves were laterally trapped because they encountered turning points and were reflected as they propagated away from the core of the anticyclonic region. Our analysis extends his one to waves that have a horizontal scale comparable to that of the jet, and 
(a)

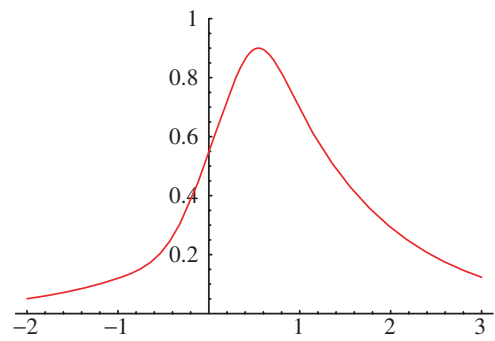

(c)

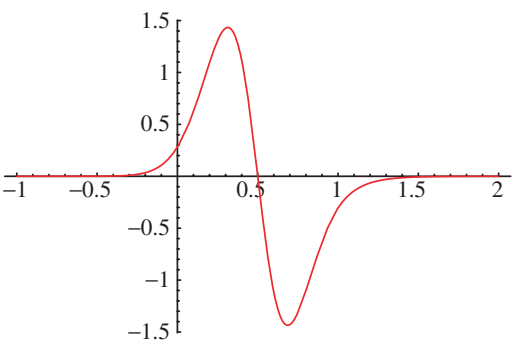

(b)

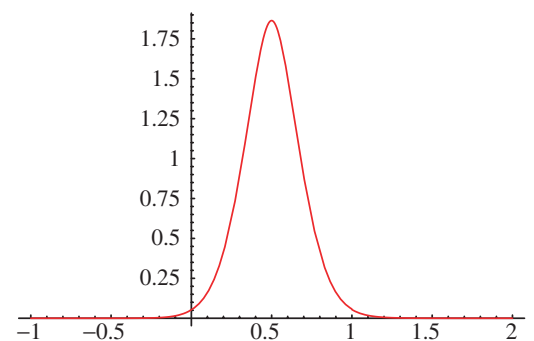

(d)

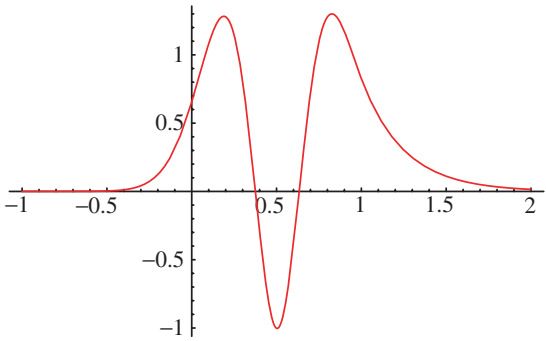

Figure 4. Horizontal structure for the trapped modes, with $B=0.5$ and $L=1$; upper-left: fundamental mode for $n=1$. The other three figures are the three possible modes for $n=6$ (upper-right $\omega=0.80$, lower-left $\omega=0.91$, and lower-right $\omega=0.98$ ). Note how increase in $n$, and to a lesser extent decrease in $\omega$, make the wave more localized within the anticyclonic part of the jet.

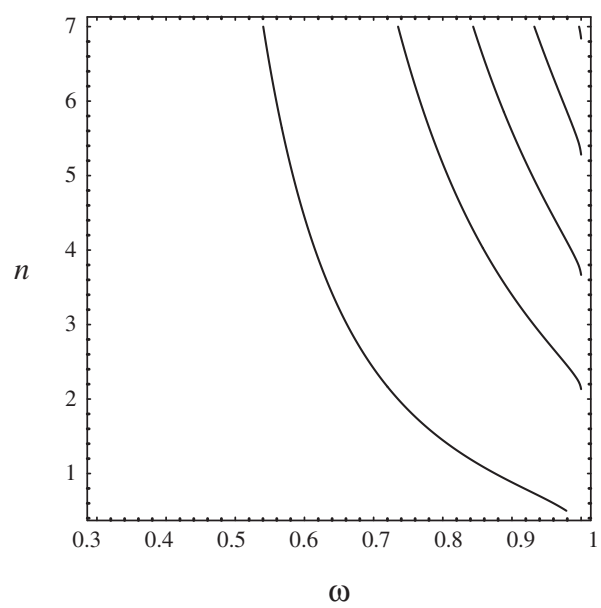

Figure 5. Dispersion relation for the trapped modes in a jet with profile given in appendix $\mathrm{B}$, with $B=0.9$ and $L=1$.

giving their full structure in the horizontal, including their decay outside of the anticyclonic region. The implications of the horizontal structure of the waves for data analysis is discussed in appendix C.2. The value we obtain for the minimal frequency of the subinertial waves also extends that found by Kunze, which was the linear approximation of (55), i.e. $\operatorname{Min}\left(1+(1 / 2) \Phi^{\prime \prime}\right)$. 
For a jet with relative vorticity lower than the opposite of the planetary vorticity $\left(-1\right.$ for our non-dimensional variables, $-f$ otherwise) unstable modes (with $\omega^{2}<0$ ) are possible. For a fluid on the $f$-plane this is the criterion for inertial (symmetric) instability, and trapped modes are the stable counterparts of inertially (or more generally symmetrically) unstable modes which appear when the anticyclonic vorticity is strong enough.

\subsection{Implications of trapping for the geostrophic adjustment}

The implication for the geostrophic adjustment is that the standard scenario, with waves propagating away from the initial anomaly in a few inertial periods is to be revised. A transverse circulation $\psi^{(0)}$ corresponding to arbitrary perturbation of the balanced jet has projections both onto continuous and onto discrete parts of the spectrum:

$$
\psi^{(0)}=\sum_{n} \sin (n \pi \bar{Z})\left[\sum_{\omega} a_{n \omega} \hat{\psi}_{n \omega}(\bar{X})+\int_{\omega>1} \mathrm{~d} \omega\left(a_{n \omega+} \hat{\psi}_{n \omega+}(\bar{X})+a_{n \omega-} \hat{\psi}_{n \omega-}(\bar{X})\right) e^{\mathrm{i} \omega t}+\text { c.c. }\right] .
$$

The modes of the continuous spectrum will propagate away, but the trapped modes will remain in the anticyclonic part of the jet. Moreover, if the PV of the initial jet is non-positive definite, part of the perturbation will break down inside the jet due to inertial instability, switching on dissipation and changing the initial PV-distribution. Hence, in general, the flow will not converge to the stationary adjusted state, but, if stable, will reach a nonstationary oscillatory state ${ }^{2}$. Note however that, as becomes evident in Lagrangian formalism, the presence of trapped waves, whatever their amplitude, can not alter the adjusted state of the fluid, unless dissipative processes come into play.

It is worth mentioning that on the example of the barotropic jet we see both an illustration of the role of the positivity of PV (inertial instability happens when PV of the initial jet (50) is non-positive) and that of the vertical boundaries (trapped modes bounce between them in the barotropic jet) in deviations of the adjustment process from the standard scenario.

The trapped waves were discussed above in the Lagrangian framework; it is worthwhile to investigate how the presence of the trapped waves manifests itself in the Eulerian framework, how they affect the classical identification of the balanced part of a flow by inversion of the Eulerian PV distribution. This question is addressed in Appendix C.3, where it is shown that the waves have a signature of order $\mathrm{O}(\delta)$ on the instantaneous PV distribution; furthermore, it is shown that they cannot be time-filtered as they have an effect of order $\mathrm{O}\left(\delta^{2}\right)$ on the time-averaged PV distribution.

We, thus, showed how to describe analytically the trapping in the anticyclonic shear region in the specific case of a barotropic jet. In order to get indications of what goes

\footnotetext{
${ }^{2}$ The nonlinear terms due to the interaction of the wave with itself will have double frequency $2 \omega$, which may fall in the continuous spectrum of free waves. This will weakly excite free waves with frequency $2 \omega$, and hence the trapped wave may slowly leak out. This, however, would occur on timescales much longer than those usually considered for geostrophic adjustment.
} 
on in the baroclinic case, a WKB-calculation is carried out in the next section 4.4 to describe the vertical propagation of trapped waves in a jet with parameters slowly varying in the vertical.

\subsection{Laterally trapped waves in a baroclinic jet varying slowly in the vertical}

In order to describe the slow vertical variations of the jet we consider two vertical coordinates: $\bar{Z}$ for the waves, and $\mathcal{Z}$ for the jet. The horizontal profile of the jet is shown in figure 3; the two parameters defining this profile, at each level $\mathcal{Z}$, are $B$, the maximum of the relative vorticity, and $L$, the half-width of the jet. $B=$ $B_{0} \exp \left(-\mathcal{Z}^{2} / 8\right)$ and for simplicity $L$ was chosen constant $L=L_{0}$.

A trapped wave in a slowly varying baroclinic jet is given by

$$
\psi(\bar{X}, \bar{Z}, t)=a(\mathcal{Z}) \hat{\psi}_{m, \omega}(\bar{X}) e^{\mathrm{i} \varphi(t, \bar{Z})}+\text { c.c. },
$$

where $a$ is the amplitude, $\hat{\psi}_{m, \omega}(x)$ is obtained as the solution of equation (53) in which $n^{2} \pi^{2}$ is replaced by $m^{2}$, and the phase function $\varphi$ verifies

$$
\frac{\partial \varphi}{\partial \bar{Z}}=m, \quad \frac{\partial \varphi}{\partial t}=-\omega
$$

with

$$
\omega=\Omega(m, B, L)
$$

We consider propagation of a wave packet; at each altitude, its wavenumber and frequency verify the dispersion relation valid for the local parameters, as shown in (59), or $\omega=\Omega(m(\mathcal{Z}), B(\mathcal{Z}), L(\mathcal{Z}))$ to emphasize the $\mathcal{Z}$-dependence. By standard manipulations we obtain

$$
\frac{\mathrm{d} m}{\mathrm{~d} t}=-\frac{\partial \Omega}{\partial B} \frac{\partial B}{\partial \mathcal{Z}}-\frac{\partial \Omega}{\partial L} \frac{\partial L}{\partial \mathcal{Z}}
$$

with $\mathrm{d} / \mathrm{d} t=\partial / \partial t+C_{g_{Z}} \partial / \partial \mathcal{Z}$. A similar equation for $d \omega / d t$ shows that the wave frequency changes only if the environment changes with time. As we consider a stationary jet, $\omega$ will remain constant.

The ray-tracing procedure is restricted to following the altitude and vertical wavenumber of the trapped wave as it propagates within the jet, starting from $\mathcal{Z}=0$. For positive $m$ and $\omega$ the group velocity is negative ${ }^{3}$. As the wave packet descends, it enters regions less favorable for trapping: the lower bound of possible frequencies for the trapped modes increases. As $\omega$ remains unchanged, $m$ increases, the group velocity decreases, and the propagation of the wave packet slows down, its vertical wavelength decreasing. Figure 6 displays the vertical propagation of the wave packet, and the evolution of its wavenumber. The initial wave parameters were: $m=4, \omega=0.792$. As the wave frequency $\omega$ is lower than the inertial period, the wave can not propagate beyond the trapping threshold (cf. (55)). In the displayed

\footnotetext{
${ }^{3}$ Because the jet is symmetric with respect to $\mathcal{Z}=0$, the results are the same for the wave with a positive group velocity.
} 


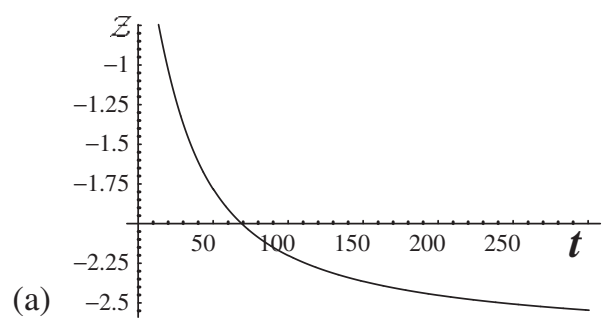

(b)

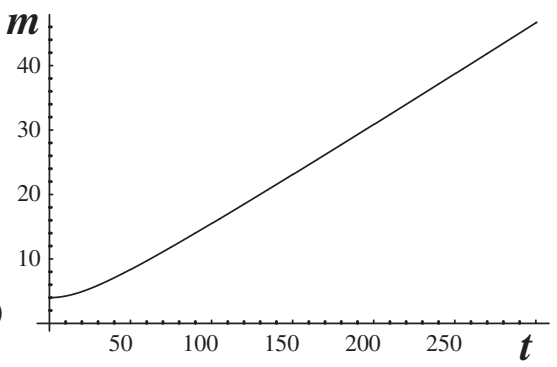

Figure 6. (a) An example of a ray; the first instants of descent (starting from $z=0$ ) are cut off; time is in inertial periods. (b) Evolution of $m$ with time.

case $\sqrt{\operatorname{Min}_{x}\left(1+\Phi^{\prime \prime}(\bar{X}, \mathcal{Z})\right)}=\omega=0.792$ gives $\mathcal{Z}=-2.8$, in agreement with the simulations. As can be seen from figure 6 , the propagation of the wave packet becomes very slow there.

Kunze, (1985) identifies this situation with a critical level. As for waves approaching a critical level the wavenumber diverges and the group velocity vanishes; note however that there is no phase velocity in the direction of the mean flow here. Obviously, the wavenumber can not grow indefinitely; the corresponding increase of gradients will lead to breaking and dissipation of the wave.

This result suggests that there are probably no trapped modes of the form $\psi(\bar{X}, \bar{Z}) \exp (\mathrm{i} \omega t)+$ c.c. in the fully baroclinic case. Indeed, whereas the WKB approximation for the propagation of a subinertial wave packet of small horizontal extent in a barotropic jet shows that the wave packet is reflected laterally, and hence laterally trapped in the anticyclonic region (Kunze, 1985), there is no counterpart of this effect in the vertical propagation: the waves are dissipated, not reflected. The vertical boundedness of the domain considered in section 4.2 is, thus, essential for the existence of the trapped modes which may bounce up and down between the vertical boundaries.

The WKB calculation also suggests that the geostrophic adjustment of a perturbation inside a jet rapidly triggers dissipative processes as subinertial waves approach their critical levels. This observation also modifies the classical scenario of adjustment, especially if one notes that no large amplitude of the initial perturbation is required for such process.

The self-consistency of the WKB approach will be broken if the term with cross derivatives (cf. (47)) $\left(\partial^{2} \phi / \partial X \partial Z\right)\left(\partial^{2} \psi / \partial X Z\right)$ becomes comparable to $\left(\partial^{2} \phi / \partial Z^{2}\right)$ $\left(\partial^{2} \phi / \partial X^{2}\right)$. Thus, for self-consistency we need to have

$$
h \gg \frac{f V L}{N^{2} H_{v}},
$$

where $H_{v}$ is the vertical scale of the jet, and $h$ is the vertical scale of the waves. For a moderate and tall jet $\left(30 \mathrm{~ms}^{-1}, 200 \mathrm{~km}\right.$ wide, $5 \mathrm{~km}$ tall $)$ in strongly stratified fluid ( $\left.f=10^{-4}, N=2 \cdot 10^{-2}\right), h$ has to be larger than $300 \mathrm{~m}$.

This constraint is somewhat restrictive, but the WKB study nevertheless provides some useful indications suggesting that the upper and lower limits of a jet act as critical 
levels for subinertial waves trapped in the anticyclonic part of the jet. Hence, they are regions where low-frequency inertia-gravity waves are likely to be observed with important amplitudes (this is consistent, for example, with numerical simulations of Klein and Tréguier (1995)), and eventually to break.

Finally, to make a link with the existence theorem for the adjusted state established earlier in the absence of vertical boundaries, the present example shows that even if the adjusted state exists, there are obstacles in reaching it starting from arbitrary initial configuration if this latter has projection on subinertial modes. This, as was just shown, will necessarily lead to switching on dissipation and deviating the adjustment process toward a different balanced state.

\section{Summary and discussion}

The use of Lagrangian variables turns out to be appropriate and efficient in the standard (in fact, semi-geostrophic) 2(1/2)-dimensional geometry of rectilinear jets and fronts in continuously stratified rotating fluid. The reason for this is that geostrophically balanced states (thermal wind) are exact steady solutions of the hydrostatic equations of motion in this geometry, and Lagrangian variables take automatically into account the advection effects which are a serious complication in Eulerian variables. The separation of the full nonlinear flow into a balanced and an unbalanced part becomes obvious, which constitutes a major advantage in studying two fundamental problems of fully nonlinear geostrophic adjustment:

1. existence and uniqueness of a smooth adjusted state with the same values of the integrals of motion as the initial state, and

2. attainability of the adjusted state through the process of emission of inertia-gravity waves from the initial perturbation.

We exploited this advantage of the Lagrangian approach to show that in the abovedefined geometry for problem 1:

- A unique adjusted state exists in the case of localized frontal anomalies with positive $\mathrm{PV}$ in infinite domain,

- Obstacles for the existence and uniqueness proof arise in the presence of boundaries, even for positive PV,

- Non-existence of a smooth adjusted state may be proved by explicit solutions of the equations of motion in the cases of zero or constant and positive PV,

- A direct relation exists between the classical semi-geostrophic deformation frontogenesis and geostrophic adjustment with no smooth final adjusted state (spontaneous frontogenesis), which allows to presume that in the presence of boundaries, strong enough initial horizontal density gradients and/or vorticities prohibit existence of a smooth adjusted state for general (non-constant) distributions of PV.

For problem 2 we obtain a prognostic Sawyer-Eliassen equation and use it to show that:

- Trapped subinertial modes may exist within jets in vertically bounded domains rendering the adjustment process incomplete, 
- These modes become unstable for jets with non positive-definite PV, giving thus a concrete physical mechanism preventing existence of the smooth adjusted state in such case,

- Subinertial modes with a horizontal scale comparable to that of the jet encounter critical layers and dissipate while vertically propagating within a baroclinic jet, which provides an alternative to the standard relaxation-by-emission adjustment mechanism, and presumably deviates relaxation from the diagnostically determined (problem 1) adjusted state.

Comparing these results with those obtained earlier by the same method in the rotating shallow water (Zeitlin et al., 2003), we see that, as expected, the adjustment in stratified fluid is substantially more complicated. In the previous case, the existence of a canonical model of wave-breaking and shock formation allowed immediate verification of the theoretical predictions by high-resolution numerical simulations (Bouchut et al., 2004). These simulations showed that although shock formation provides an effective energy sink complementary to wave emission, it does not change substantially the adjustment scenario. Wave-breaking in stratified fluid, especially with boundaries, is a much more complex process not sufficiently resolved by the existing numerical codes. Various instabilities of stratified shear flows may come into play during this process and we are far from comprehensive understanding here. That is why elucidating the role of breaking events of various nature in the adjustment of stratified fluid, especially in frontogenetic case with no smooth adjusted state, remains a challenge. As shown by our simple estimates, such cases may be rather common in the atmosphere.

\section{Acknowledgments}

We are grateful to M. Ben Jelloul for collaboration at the early stages of this work and discussions. Valuable discussions with A. Stegner are also acknowledged. R.P. was supported by ACI 'Jeunes Chercheurs' (CNRS) No. 0693. V. Z. acknowledges support from the French national program PATOM and the ACI PCN.

\section{Appendix A. A comment on the semi-geostrophic deformation frontogenesis}

The change of variables used in the classical theory of 2D semi-geostrophic (SG) deformation frontogenesis is relevant to the diagnostic 2D geostrophic adjustment problem, which is sometimes called spontaneous frontogenesis if it produces a singular adjusted state. Below we revisit the SG deformation frontogenesis (section A.1) to make it evident that it is equivalent to a succession of diagnostic geostrophic adjustment problems (section A.2).

\section{A.1. Semi-geostrophic approach to the deformation frontogenesis}

In the following, we use our notation to describe the classical approach of Hoskins and Bretherton (1972). In their study, the flow is embedded in a large scale barotropic deformation field. Hence the expression of the full horizontal velocity is 
$(-\alpha X+u, \alpha Y+v)$, where $\alpha>0$ and may vary in time. Geostrophic momentum is defined by $M=f X+v$ (only the part of the $Y$-velocity that is not associated with the deformation field is used). With a proper definition of $\phi$, the following equation is obtained:

$$
\dot{M}=-\alpha M
$$

The potential temperature and potential vorticity are conserved, so that the Lagrangian evolution of the variables $\theta, q$ and $M$ is known. Their initial distributions are $\theta_{0}, q_{0}$ and $M_{0}$.

At any time $t$ in the SG description of the 2D frontogenesis, the positions of the fluid particles $\left(X_{s g}(x, z, t), Z_{s g}(x, z, t)\right)$ are such that the thermal wind relation (5) is satisfied. The effect of the deformation field is expressed as

$$
\frac{\partial\left(X_{s g}, Z_{s g}\right)}{\partial(x, z)}=e^{-\beta}
$$

with

$$
\beta(t)=\int_{0}^{t} \alpha\left(t^{\prime}\right) \mathrm{d} t^{\prime}
$$

As noted by Hoskins and Bretherton (1972), time enters the problem only as a parameter. Hence to determine the state of the fluid at time $t$ in the semi-geostrophic description of frontogenesis, one has to solve the following equations

$$
\begin{aligned}
f \frac{\partial\left(X_{s g}, M\right)}{\partial(x, z)} & =\frac{g}{\theta_{r}} \frac{\partial\left(\theta, Z_{s g}\right)}{\partial(x, z)}, \\
\frac{\partial X_{s g}, Z_{s g}}{\partial(x, z)} & =e^{-\beta}
\end{aligned}
$$

with boundary conditions

$$
Z_{s g}(x, 0)=0 \quad \text { and } \quad Z_{s g}(x, H)=H,
$$

and where $\theta$ and $M$ are given by

$$
\theta(x, z, t)=\theta_{0}(x, z) \quad \text { and } \quad M(x, z, t)=e^{-\beta} M_{0}(x, z) .
$$

It is easy to see from (A.3b) and (A.5) that PV (cf. (4)) is conserved.

\section{A.2. Equivalent geostrophic adjustment problem}

The above problem has a form nearly identical to the diagnostic adjustment, as presented in section 3. By rescaling the $x$ variable, we now show that, indeed, the two problems are equivalent. 
We rescale $x: s=x \exp (-\beta)$, which would be the position at $t$ of a particle initially at $x$ if only the deformation field were present. Equations (A.3)-(A.5) are then identical to (10), (11), with the following initial distributions for $\theta$ and $M$ :

$$
\theta_{I}(s, z)=\theta_{0}\left(s e^{\beta}, z\right) \quad \text { and } \quad M_{I}(s, z)=e^{-\beta} M_{0}\left(s e^{\beta}, z\right)
$$

It is worth noting that the SG equations are an approximation, whereas the equations $(10 \mathrm{a}, \mathrm{b})$ for the adjustment problem are exact. The hypothesis rendering the two equivalent is that $\mathrm{HB}$ suppose that at any $t$, the $Y$-velocity is nearly in geostrophic balance; their expression for PV only takes into account the geostrophic part of $v$, and therefore is the same as the exact expression for the PV in a stationary adjusted solution of the full equations.

Although the two problems are formally equivalent, it seems that it was not realized in previous studies of the geostrophic adjustment of frontal anomalies. In particular, the description by Kalashnik (1998) of discontinuities penetrating the fluid parallels that of discontinuities introduced by Cullen (1983) and Cullen and Purser (1984) to extend the validity of the semi-geostrophic description of frontogenesis. The principles used are the same: conservations of Lagrangian quantities, and the Margules relation relating the jumps of $M$ and $\theta$ at the discontinuity.

An immediate consequence is that, as discontinuities are known to form in SG frontogenesis for any initial condition with a horizontal potential temperature gradient if the deformation field acts for long enough time, there always exist initial conditions for which it is impossible to reach a continuous steady state without dissipation and the adjusted state is not well-defined. Another consequence is that examples available in the literature on SG deformation frontogenesis can be interpreted as illustrations of adjusted states for geostrophic adjustment problems with the appropriate initial conditions.

\section{Appendix B. An explicit example of trapped modes in a barotropic jet}

We here construct explicit analytical solutions of the trapped waves considered in section 4.2 for the special case of baratropic jets having piecewise linear relative vorticity. The relative vorticity $\Phi^{\prime \prime}$ is symmetric and defined by using two parameters: $L$, the half-width of the jet, and $B$, its maximum value. It is given by a linear function in each of the five regions denoted as follows: I for $\bar{X}<-L$, II for $-L<\bar{X}<-L / 2$, III for $-L / 2<\bar{X}<L / 2$, IV for $L / 2<\bar{X}<L$, and V for $L<\bar{X}$.

The geopotential is (cf. figure 4 ):

$$
\begin{aligned}
& \Phi(\bar{X})=0 \quad \text { in I, } \\
& \Phi(\bar{X})=\frac{B L^{2}}{3}\left(\frac{\bar{X}}{L}+1\right)^{3} \quad \text { in II, } \\
& \Phi(\bar{X})=B L^{2}\left(-\frac{\bar{X}^{3}}{3 L^{3}}+\frac{1}{2} \frac{\bar{X}}{L}+\frac{1}{4}\right) \text { in III, }
\end{aligned}
$$




$$
\begin{aligned}
& \Phi(\bar{X})=\frac{B L^{2}}{3}\left(\left(\frac{\bar{X}}{L}+1\right)^{3}+\frac{3}{2}\right) \quad \text { in IV, } \\
& \Phi(\bar{X})=\frac{B L^{2}}{2} \text { in } \mathrm{V},
\end{aligned}
$$

and the relative vorticity $\Phi^{\prime \prime}$ and $v^{(0)}=\Phi^{\prime}$ easily follow. In each of the five regions, we have an equation of the form (cf. (44))

$$
f^{\prime \prime}+(c+a s) f=0
$$

with solutions

$$
f(s)=A \mathcal{A}\left(\frac{c+a s}{a^{2 / 3}}\right)+B \mathcal{B}\left(\frac{c+a s}{a^{2 / 3}}\right),
$$

where $\mathcal{A}$ and $\mathcal{B}$ are the Airy functions. Hence, equations for the horizontal structure of a trapped mode of frequency $\omega$ and its solutions will be, in each of the five zones:

I. $\psi_{1}^{\prime \prime}-c \psi_{1}=0$,

$$
\psi_{1}(s)=A_{1} e^{\sqrt{c s}}+B_{1} e^{-\sqrt{c} s},
$$

II. $\psi_{2}^{\prime \prime}-\left(c+\gamma^{3}(s+L)\right) \psi_{2}=0$,

$$
\psi_{2}(s)=A_{2} \mathcal{A}\left(\frac{c+\gamma^{3}(s+L)}{\gamma^{2}}\right)+B_{2} \mathcal{B}\left(\frac{c+\gamma^{3}(s+L)}{\gamma^{2}}\right),
$$

III. $\psi_{3}^{\prime \prime}-\left(c-\gamma^{3} s\right) \psi_{3}=0$,

$$
\psi_{3}(s)=A_{3} \mathcal{A}\left(\frac{c-\gamma^{3} s}{\gamma^{2}}\right)+B_{3} \mathcal{B}\left(\frac{c-\gamma^{3} s}{\gamma^{2}}\right)
$$

IV. $\psi_{4}^{\prime \prime}-\left[c+\gamma^{3}(s-L)\right] \psi_{4}=0$,

$$
\psi_{4}(s)=A_{4} \mathcal{A}\left(\frac{c+\gamma^{3}(s-L)}{\gamma^{2}}\right)+B_{4} \mathcal{B}\left(\frac{c+\gamma^{3}(s-L)}{\gamma^{2}}\right),
$$

V. $\psi_{5}^{\prime \prime}-c \psi_{5}=0$,

$$
\psi_{5}(s)=A_{5} e^{-\sqrt{c} s}+B_{5} e^{\sqrt{c s}},
$$

where $c=1-\omega^{2}$ and $\gamma=(2 B / L)^{1 / 3}$.

At each of the four boundaries between two regions, the functions and their first derivative must match. Thus, matching at point $s=-L$ yields two linear equations for the two unknowns $A_{2}$ and $B_{2}$ to be expressed using $A_{1}$ and $B_{1}$. Similarly, matching 
at the other points will give expressions for each pair $A_{i}, B_{i}$ using coefficients $A_{i-1}, B_{i-1}$ :

$$
\begin{aligned}
\text { I-II : } \quad A_{2} & =A_{1} \frac{e^{-\sqrt{c} L}}{\mathcal{C C}}\left(\mathcal{B}^{\prime}\left(p_{1}\right)-\frac{\sqrt{c}}{\gamma} \mathcal{B}\left(p_{1}\right)\right), \\
B_{2} & =A_{1} \frac{e^{-\sqrt{c} L}}{\mathcal{C C}}\left(\frac{\sqrt{c}}{\gamma} \mathcal{A}\left(p_{1}\right)-\mathcal{A}^{\prime}\left(p_{1}\right)\right), \\
\text { II-III : } \quad A_{3} & =\frac{1}{\mathcal{C C}}\left[(\mathcal{A B})^{\prime}\left(p_{2}\right) A_{2}+\left(\mathcal{B}^{2}\right)^{\prime}\left(p_{2}\right) B_{2}\right], \\
B_{3} & =-\frac{1}{\mathcal{C C}}\left[\left(\mathcal{A}^{2}\right)^{\prime}\left(p_{2}\right) A_{2}+(\mathcal{A B})^{\prime}\left(p_{2}\right) B_{2}\right], \\
\text { III-IV : } \quad A_{4} & =\frac{1}{\mathcal{C C}}\left[(\mathcal{A B})^{\prime}\left(p_{3}\right) A_{3}+\left(\mathcal{B}^{2}\right)^{\prime}\left(p_{3}\right) B_{3}\right], \\
B_{4} & =-\frac{1}{\mathcal{C C}}\left[\left(\mathcal{A}^{2}\right)^{\prime}\left(p_{3}\right) A_{3}+(\mathcal{A B})^{\prime}\left(p_{3}\right) B_{3}\right], \\
\text { IV-V : } \quad A_{5} & =\frac{e^{\sqrt{c} L}}{2}\left[\left(\mathcal{A}\left(p_{1}\right)-\frac{\gamma}{\sqrt{c}} \mathcal{A}^{\prime}\left(p_{1}\right)\right) A_{4}+\left(\mathcal{B}\left(p_{1}\right)-\frac{\gamma}{\sqrt{c}} \mathcal{B}^{\prime}\left(p_{1}\right)\right) B_{4}\right], \\
B_{5} & =\frac{e^{-\sqrt{c} L}}{2}\left[\left(\mathcal{A}\left(p_{1}\right)+\frac{\gamma}{\sqrt{c}} \mathcal{A}^{\prime}\left(p_{1}\right)\right) A_{4}+\left(\mathcal{B}\left(p_{1}\right)+\frac{\gamma}{\sqrt{c}} \mathcal{B}^{\prime}\left(p_{1}\right)\right) B_{4}\right],
\end{aligned}
$$

where

$$
\begin{aligned}
\mathcal{C} C & =\mathcal{A}(s) \mathcal{B}^{\prime}(s)-\mathcal{A}^{\prime}(s) \mathcal{B}(s)=\text { constant }, \\
p_{1} & =\frac{c}{\gamma^{2}}, \quad p_{2}=\frac{c+B}{\gamma^{2}}, \quad p_{3}=\frac{c-B}{\gamma^{2}} .
\end{aligned}
$$

A full solution represents a trapped mode if it vanishes outside the jet region, i.e. if $B_{1}=0$ and $B_{5}=0$. Imposing $B_{1}=0$ (B.5a-d) then give an expression for $B_{5}$ proportional to $A_{1}$, the proportionality factor being a function of $c, B$ and $L\left(\gamma=(2 B / L)^{1 / 3}\right)$. Using (B.5) this factor can be written as

Cond $(c, B, L)$

$$
\begin{aligned}
= & \left(\sqrt{c} \mathcal{A}\left(p_{1}\right)+\gamma \mathcal{A}^{\prime}\left(p_{1}\right)\right)\left[\left((\mathcal{A})^{\prime}\left(p_{3}\right)(\mathcal{A})^{\prime}\left(p_{2}\right)-\left(\mathcal{B}^{2}\right)^{\prime}\left(p_{3}\right)\left(\mathcal{A}^{2}\right)^{\prime}\left(p_{2}\right)\right)\left(\mathcal{B}^{\prime}\left(p_{1}\right)-\frac{\sqrt{c}}{\gamma^{2}} \mathcal{B}\left(p_{1}\right)\right)\right. \\
& \left.+\left((\mathcal{A B})^{\prime}\left(p_{3}\right)\left(\mathcal{B}^{2}\right)^{\prime}\left(p_{2}\right)-\left(\mathcal{B}^{2}\right)^{\prime}\left(p_{3}\right)(\mathcal{A B})^{\prime}\left(p_{2}\right)\right)\left(\frac{\sqrt{c}}{\gamma^{2}} \mathcal{A}\left(p_{1}\right)-\mathcal{A}^{\prime}\left(p_{1}\right)\right)\right] \\
& -\left(\sqrt{c} \mathcal{B}\left(p_{1}\right)+\gamma \mathcal{B}^{\prime}\left(p_{1}\right)\right)\left[\left(\left(\mathcal{A}^{2}\right)^{\prime}\left(p_{3}\right)(\mathcal{A B})^{\prime}\left(p_{2}\right)-(\mathcal{A B})^{\prime}\left(p_{3}\right)\left(\mathcal{A}^{2}\right)^{\prime}\left(p_{2}\right)\right)\left(\mathcal{B}^{\prime}\left(p_{1}\right)-\frac{\sqrt{c}}{\gamma^{2}} \mathcal{B}\left(p_{1}\right)\right)\right. \\
& \left.\left.+\left(\mathcal{A}^{2}\right)^{\prime}\left(p_{3}\right)\left(\mathcal{B}^{2}\right)^{\prime}\left(p_{2}\right)-(\mathcal{A B})^{\prime}\left(p_{3}\right)(\mathcal{A B})^{\prime}\left(p_{2}\right)\right)\left(\frac{\sqrt{c}}{\gamma^{2}} \mathcal{A}\left(p_{1}\right)-\mathcal{A}^{\prime}\left(p_{1}\right)\right)\right] .
\end{aligned}
$$


The dependencies on $c, B$ and $L$ are hidden in this equation inside $p_{1}, p_{2}$ and $p_{3}$ (cf. (B.6)), and $\gamma$. The frequency of the trapped mode enters via $c=1-\omega^{2}$. The jet with parameters $B, L$ has a trapped mode of frequency $\omega$ if $\operatorname{Cond}\left(1-\omega^{2}, B, L\right)$ is zero whence the frequencies of the trapped modes follow (cf. figure 5).

\section{Appendix C. Remarks on the trapped modes in the Eulerian framework}

The trapped modes have been studied above in the Lagrangian framework, which is more convenient in the adjustment context. As it is more common (often unavoidable) to consider flows from the Eulerian viewpoint, it is worth considering the manifestations of the trapped modes in the Eulerian framework. Below we concentrate on two specific aspects: the manifestation of trapped modes in data, such as radiosoundings (C.1), (C.2) and the effect of the trapped waves on PV (C.3).

\section{C.1. Trapped modes in the Eulerian framework}

From the Eulerian perspective, the independent variables are $(X, Z, t)$. We consider a barotropic jet plus a single trapped mode. The total geopotential is $\Phi(X)+Z^{2} / 2+\phi$. The total velocity in the $Y$ direction is $\Phi^{\prime}+v$, where the variables $u, v$ and $\phi$ correspond to the wave perturbation. The equation for the structure of the trapped modes has the same form as (47) in Eulerian coordinates, but $\psi^{(0)}$ is now a streamfunction for $u^{(0)}=-\partial \psi^{(0)} / \partial Z$ and $w^{(0)}=\partial \psi^{(0)} / \partial X$ :

$$
\left(1+\Phi^{\prime \prime}+\frac{\partial^{2}}{\partial t^{2}}\right) \frac{\partial^{2} \psi^{(0)}}{\partial Z^{2}}+\frac{\partial^{2} \psi^{(0)}}{\partial X^{2}}=0
$$

For a single mode the perturbation velocity is

$$
u^{(0)}=-a \cos (n \pi Z) n \pi \hat{\psi}_{n \omega}(X) \cos (\omega t+\varphi) .
$$

From the momentum equation in the $Y$ direction

$$
\frac{\partial v^{(0)}}{\partial t}+\left(1+\Phi^{\prime \prime}\right) u^{(0)}=0,
$$

we obtain

$$
v^{(0)}=a \cos (n \pi Z) \frac{n \pi}{\omega}\left(1+\Phi^{\prime \prime}\right) \hat{\psi}_{n \omega}(X) \sin (\omega t+\varphi) .
$$

From the conservation of potential temperature, $\phi^{(0)}$ can be derived:

$$
\phi^{(0)}=-a \cos (n \pi Z) \frac{1}{n \pi \omega} \hat{\psi}_{n \omega}^{\prime}(X) \sin (\omega t+\varphi) .
$$




\section{C.2. Consequences of the spatial structure of the trapped modes for data analysis}

The question we ask below is that of signature of trapped waves in data, e.g. in vertical profiles of the velocity perturbation obtained from radiosoundings or from radar measurements. The characteristics of inertia-gravity waves in such observations are retrieved from the hodograph analysis (e.g. Hirota and Niki, 1985); in particular, the orientation of the ellipse in the hodograph plane indicates the orientation of the wave-vector: for waves propagating transverse to the jet direction, we expect their hodographs to display a correspondingly oriented ellipse with the aspect ratio equal to $f / \omega(1 / \omega$ in our scaled expressions).

As can be seen from (C.2) and (C.4), the variations in time of the perturbations of $u$ and $v$ due to a wave in the jet will give ellipses with an aspect ratio $\left(1+\Phi^{\prime \prime}\right) / \omega$ that varies in the cross-front direction. Where the trapped mode is strongest (i.e. where $\Phi^{\prime \prime}<0$ ), it will have the expected transverse orientation. In the rest of the domain however, and on the borders of the anicyclonic region, where the wave is weaker but may nevertheless be detected, its signature in the hodograph will have an unusual alongfront orientation. The above analysis suggests that for large scale waves of low frequency in a jet region, one should proceed with special care while interpreting the hodograph data.

\section{C.3. Trapped waves and the Eulerian distribution of $P V$}

We focus here on the effect of the trapped modes on the PV because this variable is often used to diagnose, by inversion, the balanced part of the flow, and because analysis of waves over the rest-state suggests that IGW have no PV-signature.

The expression for the PV is

$$
q=\left(1+\Phi^{\prime \prime}+\frac{\partial v}{\partial X}\right)\left(1+\frac{\partial^{2}}{\partial Z^{2}}\right)-\frac{\partial v}{\partial Z} \frac{\partial^{2} \phi}{\partial X \partial Z}
$$

Therefore, $q=q_{b}+\delta q^{(1)}+\delta^{2} q^{(2)}$ with

$$
\begin{aligned}
q_{b} & =\left(1+\Phi^{\prime \prime}\right) \\
q^{(1)} & =\left(1+\Phi^{\prime \prime}\right) \frac{\partial^{2} \phi^{(0)}}{\partial Z^{2}}+\frac{\partial v^{(0)}}{\partial X} \\
q^{(2)} & =\left(1+\Phi^{\prime \prime}\right) \frac{\partial^{2} \phi^{(1)}}{\partial Z^{2}}+\frac{\partial v^{(1)}}{\partial X}+\frac{\partial v^{(0)}}{\partial X} \frac{\partial^{2} \phi^{(0)}}{\partial Z^{2}}-\frac{\partial v^{(0)}}{\partial Z} \frac{\partial^{2} \phi^{(0)}}{\partial X \partial Z} .
\end{aligned}
$$

Substituting (C4) and (C5) into (C6b) yields

$$
q^{(1)}=a \cos (n \pi Z) \frac{n \pi}{\omega}\left(2\left(1+\Phi^{\prime \prime}\right) \hat{\psi}_{n \omega}^{\prime}+\Phi^{\prime \prime \prime} \hat{\psi}_{n \omega}\right) \sin (\omega t+\varphi) .
$$

Hence, the waves have a signature of $\operatorname{order} \delta$, i.e. of the order of their amplitude, in the instantaneous distribution of PV. This is due to the $\mathrm{O}(1)$ gradients of PV in the advection terms. An example of this phenomenon in a three-dimensional situation is given in figure 11 of the numerical study by O'Sullivan and Dunkerton (1995) of 
the generation of inertia-gravity waves during the life-cycle of an unstable baroclinic wave. Because of advection of PV by waves, separating the flow by the inversion of PV will yield a 'balanced' part that varies on the same timescale as the wave, as was obtained in the shallow water model by Kuo and Polvani (1999).

This $\mathrm{O}(\delta)$ contribution, however, disappears when a time-average is applied as can be seen from (C.7). Nevertheless, at the next order the two terms involving $v^{(0)}$ and $\phi^{(0)}$ give wave-wave contributions that are non-zero when averaged over time:

$$
\phi \frac{\partial \tilde{v}^{(0)}}{\partial X} \frac{\partial \tilde{\phi}^{(0)}}{\partial Z^{2}}-\frac{\partial \tilde{v}^{(0)}}{\partial Z} \frac{\partial \tilde{\phi}^{(0)}}{\partial X \partial Z}=-\frac{1}{2} a^{2} n^{2} \pi^{2}\left[\hat{v}_{n \omega}^{\prime} \hat{\phi}_{n \omega} \cos ^{2}(n \pi Z)+\hat{v}_{n \omega} \hat{\phi}_{n \omega}^{\prime} \sin ^{2}(n \pi Z)\right] .
$$

Hence, the waves not only have an instantaneous $\mathrm{O}(\delta)$ signature in the Eulerian distribution of $\mathrm{PV}$, but they also have a $\mathrm{O}\left(\delta^{2}\right)$ signature in the time-averaged distribution of PV, despite the unambiguous separation between the timescales of the waves and of the balanced (stationary) flow in this configuration.

\section{References}

Bennetts, D.A. and Hoskins, B.J., Conditional symmetric instability-possible explanation for frontal rainbands. Q.J.R. Meteorol. Soc., 1979, 105, 945-962.

Blumen, W. and Williams, R.T., Unbalanced frontogenesis: Part I, zero potential vorticity. J. Atmos. Sci., 2001, 58, 2180-2195.

Blumen, W. and $\mathrm{Wu}, \mathrm{R}$., Geostrophic adjustment: frontogenesis and energy conversion. J. Phys. Oceanogr., 1995, 25, 428-438.

Bouchut, F., Sommer, Le, J. and Zeitlin, V., 1D rotating shallow water: nonlinear semi-geostrophic adjustment, slow manifold and nonlinear wave phenomena. Part 2. High-resolution numerical simulations. J. Fluid Mech., 2004, 514, 35-63.

Cho, H.-R., Shepherd, T.G. and Vladimirov, V.A., Application of the direct Lyapunov method to the problem of symmetric stability in the atmosphere. J. Atmos. Sci., 1993, 50, 822-835.

Csanady, G.T. On the equilibrium shape of the thermocline in a shore zone. J. Phys. Oceanogr., 1971, 1, 263-270.

Cullen, M.J.P. Solutions to a model of a front forced by deformation. Q.J.R. Meteorol. Soc., 1983, 109, $565-573$.

Cullen, M.J.P. and Purser, R.J., An extended Lagrangian theory of semi-geostrophic frontogenesis. J. Atmos. Sci., 41, 1984, 1477-1497.

Cullen, M.J.P. and Purser, R.J., Properties of the Lagrangian semigeostrophic equations. J. Atmos. Sci., 1989, 46, 2684-2697.

Cullen, M.J.P., Norbury, J. and Purser, R.J., Generalized Lagrangian solutions for atmospheric and oceanic flows. SIAM J. Appl. Math., 1991, 51, 20-31.

Fritts, D.C. and Luo, Z., Gravity wave excitation by geostrophic adjustment of the jet stream. Part I: Two-dimensional forcing. J. Atmos. Sci., 1992, 49, 681-697.

Gill, A.E. Atmosphere-ocean Dynamics. Volume 30 of the International Geophysics Series, 1982, Academic Press.

Hirota, I. and Niki, T., A statistical study of inertia-gravity waves in the middle atmosphere. J. Meteor. Soc. Japan, 1985, 63, 1055-1065.

Holton, J.R., An introduction to Dynamic Meteorology. third edn. 1992, Academic Press.

Hoskins, B.J. and Bretherton, F.P., Atmospheric frontogenesis models: mathematical formulation and solution. J. Atmos. Sci., 1972, 29, 11-37.

Hoskins, B.J., McIntyre, M.E. and Robertson, A.W., On the use of isotropic potential vorticity maps. Q.J.R. Meteorol. Soc., 1985, 11, 877-946.

Kalashnik, M.V., Forming of frontal zones during geostrophic adjustment in a continuously stratied .uid. Izvetiya, Atmospheric and Oceanic Physics, 1998, 34, 785-792.

Kalashnik, M.V., Geostrophic adjustment and frontogenesis in a continuously stratified fluid. Izvetiya, Atmospheric and Oceanic Physics, 2000, 36, 386-395.

Klein, P. and Tréguier, A.M., Dispersion of wind-induced inertial waves by a barotropic jet. J. Mar. Res., 1995, 53, 1-22. 
Kunze, E., Near-inertial wave propagation in geostrophic shear. J. Phys. Oceanogr., 1985, 15, 544-565.

Kunze, E. and Boss, E., A model for vortextrapped internal waves. J. Phys. Oceanogr., 1998, 28, $2104-2115$.

Kuo, A.C. and Polvani, L.M., Time-dependent fully nonlinear geostrophic adjustment. J. Phys. Oceanogr., 1997, 27, 1614-1634.

Kuo, A.C. and Polvani, L.M., Wave-vortex interaction in rotating shallow water. Part 1. One space dimension. J. Fluid Mech., 1999, 394, 1-27.

Laurentiev, M.A. and Shabat, B.V., Methods of the Theory of Functions of a Complex Variable. Fifth edn. 1987, Nauka, Moscow (in Russian).

Lions, P.-L., Trudinger, N.S. and Urbas, J.E., The Neumann problem for equations of Monge-Ampére type. Comm. Pure Appl. Math, 1986, 39, 539-563.

Llewllyn-Smith, S.G., Near inertial oscillation of a barotropic vortex: trapped modes and time evolution. J. Phys. Oceanogr., 1999, 29, 747-761.

McWilliams, J.C. and Gent, P.R., Intermediate models of planetary circulations in the atmosphere and ocean. J. Atmos. Sci., 1980, 37, 1657-1678.

Messiah, A., 1995, Mécanique Quantique. Tome 1, new edition, Dunod, Paris (in French).

Ou, Hsien Wang, Geostrophic adjustment: a mechanism for frontogenesis. J. Phys. Oceanogr., 1984, 14, 994-1000.

Pogorelov, A.V., Extrinsic Geometry of Convex Surfaces. 1973, American Mathematical Society.

Rossby, G.G., On the mutual adjustment of pressure and velocity distributions in certain simple current systems II. J. Mar. Res., 1938, 1, 239-263.

Shutts, G.J. and Cullen, M.J.P., Parcel stability and its relation to semigeostrophic theory. J. Atmos. Sci., 1987, 44, 1318-1330.

Urbas, J., Oblique boundary value problems for equations of Monge-Ampére type. Calc. Var., 1998, 7, 19-39.

van Haren, H., On the polarization of oscillatory currents in the Bay of Biscay. J. Geophys. Res., 2003, 108(C9), 3290.

$\mathrm{Wu}, \mathrm{R}$. and Blumen, W., Geostrophic adjustment of a zero potential vorticity .ow initiated by a mass imbalance. J. Phys. Oceanogr., 1995, 25, 439-445.

Xing, J. and Davies, A.M., On the influence of a surface coastal front on near-inertial wind-induced internal wave generation. J. Geophys. Res., 2004, 109, C01023.

Zeitlin, V., Medvedev, S.B. and Plougonven, R., Frontal geostrophic adjustment, slow manifold and nonlinear wave phenomena in one-dimensional rotating shallow-water. Part 1: Theory. J. Fluid Mech., 2003, 481, 269-290. 